\title{
A case study in programming a quantum annealer for hard operational planning problems
}

\author{
Eleanor G. Rieffel, Davide Venturelli, Bryan O'Gorman, Minh B. Do, Elicia Prystay, and Vadim N. Smelyanskiy \\ QuAIL, NASA Ames Research Center, Moffett Field, CA 94035
}

\begin{abstract}
We report on a case study in programming an early quantum annealer to attack optimization problems related to operational planning. While a number of studies have looked at the performance of quantum annealers on problems native to their architecture, and others have examined performance of select problems stemming from an application area, ours is one of the first studies of a quantum annealer's performance on parametrized families of hard problems from a practical domain. We explore two different general mappings of planning problems to quadratic unconstrained binary optimization (QUBO) problems, and apply them to two parametrized families of planning problems, navigation-type and scheduling-type. We also examine two more compact, but problemtype specific, mappings to QUBO, one for the navigation-type planning problems and one for the scheduling-type planning problems. We study embedding properties and parameter setting, and examine their effect on the efficiency with which the quantum annealer solves these problems. From these results we derive insights useful for the programming and design of future quantum annealers: problem choice, the mapping used, the properties of the embedding, and the annealing profile all matter, each significantly affecting the performance.
\end{abstract}

\section{INTRODUCTION}

Quantum computing has been proven to provide more efficient means for solving certain classes of specialized problems than is possible classically, and in other cases is strongly suspected to do so [31, 37]. There are other classes of problems, however, for which quantum computing does not provide an advantage. One of the biggest open questions in quantum computing is the breadth of its applications. Many of the most complex computations carried out in the practical world today use heuristic algorithms which have not been mathematically proven to outperform other approaches, but have been shown to be more effective empirically. Quantum heuristic algorithms exist, but it is only when quantum computational devices that can carry out these algorithms become available that we can learn whether they are more effective than current classical approaches. The most prominent quantum heuristic is quantum annealing.

Quantum annealing [16, 18, 24, 42] is a metaheuristic for solving optimization problems which bears some resemblance to simulated annealing, a classical metaheuristic. It works by starting the system in the ground state of a known, easy-to-implement Hamiltonian $H_{I}$ and gradually varying the Hamiltonian until it becomes a Hamiltonian $H_{P}$ that encodes the cost function for the problem at hand:

$$
H(s)=A(s) H_{I}+B(s) H_{P},
$$

where $s \in[0,1], A(0)=1=B(1)$, and $A(1)=0=B(0)$. The intuition behind quantum annealing is that it explores the cost-function landscape, but has means of exploration not open to classical methods such as quantum tunneling.

Within the last couple of years, D-Wave quantum annealers have become available. While debate continues as to the extent to which the D-Wave machine is quantum [7, 8, 23, 40, 41, 43, 45, 46, these machines provide the first opportunity for researchers to experiment with quantum annealing. Quantum computational hardware is maturing to the point that a number of different quantum computational devices implementing specialized algorithms such as quantum annealing will become available in the next several years.

Because most physical interactions are 2-local (i.e. pairwise), most emerging quantum technologies will support only 2-local interactions. For this reason, the problem Hamiltonian $H_{P}$ should be Ising, containing only 2local terms between the qubits. A standard translation maps between problem Hamiltonians on qubits and cost functions of binary variables. When translated to a cost function, the 2-local condition on the problem Hamiltionian means that the cost function must be a Quadratic Unconstrained Binary Optimization (QUBO) problem. Furthermore, limitations on the ability to couple a single qubit to more than a few other qubits, means that variables that appear in many terms will need to be represented by multiple physical qubits in order to enable the required connections. For example, the D-Wave processors use a Chimera architecture in which each qubit is connected to at most 6 other qubits (See Fig. 13), so any logical qubit in problem Hamiltonian that appears with more than 6 other logical qubits must be represented by multiple physical qubits in order for the problem to be expressible in this architecture. The first step, obtaining the QUBO, is referred to as mapping the problem to QUBO. The second step is referred to as embedding the QUBO in the hardware. Problems that fit directly on the machine, so do not require an embedding step, are referred to as native problems.

Given a specific quantum annealing hardware architecture, there are three high-level research challenges:

- Problem design: Identify potential applications with appropriately difficult combinatorial optimization problems; extract core aspects of these 
problems that contribute to their difficulty; generate families of benchmark problems that are small enough to be run on the newly available devices, but are nevertheless interesting in spite of their smallness.

- Mapping to QUBO: Design general approaches to map these problems to QUBO problems, and to make good choices of parameters, such as the relative weighting of QUBO terms.

- Embedding in hardware: Determine which physical qubits should represent each logical qubit, the strength of the internal couplings (between qubits representing the same logical qubit), and how to distribute the external couplings (between sets of qubits representing coupled logical qubits). Thus, there are two aspects of embedding: the topological aspect and the parameter-setting aspect.

In future architectures, there will be additional research challenges, such as making good choices for the annealing time and the annealing profile (the functions $A(s)$ and $B(s)$ determining the weighting of the driver Hamiltonian and the problem Hamiltonian throughout the run).

A previous paper 38 focused on the design of two parametrized families of hard planning problems, navigation-type and scheduling-type, that capture aspects common to many real-world planning problems, exhibit exponential scaling in hardness even at small sizes, and provide insights into state-of-the-art planning algorithms. These problems can be used to investigate novel approaches to planning problems, as we do here for quantum annealing. Since these problems are optimization versions of NP-complete problems, we expect any approach, whether quantum or classical, to scale exponentially with problem size. In the classical case, the slope of that exponential can be radically different from algorithm to algorithm, and we expect the same to be true for quantum heuristics. Some quantum heuristics will be better than others, and there is the exciting possibility that quantum approaches could outperform classical heuristics on these or other hard combinatorial optimization problems. Such an algorithm would have significant practical impact.

This paper focuses on the second and third programming challenges, mapping and embedding, for hard combinatorial optimization problems that arise in operational planning. We explore multiple ways of mapping planning problems to QUBO problems, explore embeddings of these problems, and provide comparisons of the effectiveness of the DWave Two machine housed at NASA Ames in solving these problems under different mappings and parameter choices. In particular, we examine two different approaches for mapping general planning problems to QUBO problems, and apply both of these mappings to the navigation-type family and the scheduling-type family of planning problems of [38. In part because we are only able to embed the smallest size problems in the DWave Two architecture under these mappings, we developed two more compact but problem-type specific embeddings, one for the navigation-type problems and one for the scheduling-type problems. Ultimately, we are interested in the general approaches, since only they would be applicable to real-world planning problems that contain aspects of both navigation and scheduling that are not easily separated. But at this early stage we can learn from the behavior of the quantum annealer on the problems obtained from the more specialized mappings.

We also explore embedding properties and parameter choices, and their relation to the efficiency with which the D-Wave Two solves these problems. For example, the internal couplings between physical qubits representing the same logical qubits must be set. Here, we look at the case in which all internal couplings have the same strength $J_{i n t}$, and investigate how the performance is affected by variations in this value. We are particularly interested in what these initial results tell us about how different future machine architectures and different programming choices could affect the ability of these machines to solve these problems.

Our work is the first to explore the programming and performance of a D-Wave machine on parametrized families of hard problems stemming from applications. A number of studies have benchmarked D-Wave performance on native problems [8, 39, 47,49]. A recent study [44 analyzes performance on families of non-native problems, random 2-dimensional Ising lattices and random Ising problems on fully connected graphs. As non-native problems, they do require embeddings, and the paper explores similar issues in that context to some we explore here such as the setting of the internal coupling constant $J_{\text {int }}$, but while these structures appear in appications, these problems do not come from a specific applications. On the other hand, a number of groups have explored applications [3, 5, 25, 32, 34, but either on set of small instances for which there is no notion of hardness or a few larger, but specific instances, rather than parametrized families of instances whose hardness is expected to scale exponentially for all approaches, and on which the performance of the best classical approaches confirms the exponentially increasing hardness with size. Very recent work [35] suggests a programmatic approach to some of the issues we discuss here, such as setting $J_{i n t}$, with examples to problems from a few different application domains.

Our main contributions include an analysis of performance on a large, parametrized set of hard benchmark problems stemming from an application domain, a comparison of the effectiveness of different mappings of these problems to QUBO, an investigation of embedding properties and parameter setting and their effect on performance, and an evaluation of future architectures in light of these findings. We derive from this study insights useful for the programming and design of future quantum annealers. Specifically, 
- scheduling-type planning problems are more amenable to near-term quantum annealing approaches than navigation-type planning problems, which are difficult to embed in the hardware,

- the choice of QUBO mapping makes a marked difference in the success of an annealing algorithm even when the QUBO sizes are similar,

- embedding metrics beyond embedding size and maximum component size are needed in order to predict and optimize performance,

- increasing the size of the unit cells of the Chimera architecture (Fig. 13), and thereby the local connectivity, would much more significantly impact our ability to run instances from applications than would simply increasing the number of unit cells, and

- support for different annealing profiles (weightings of the driver and problem Hamiltonians throughout the run) need to be supported and could potentially lead to significantly improved results.

We first provide a brief review of classical planning (Sec. II), and then describe the parametrized families of hard navigation-type and scheduling-type planning problems used in our experiments in Secs. [II] and IV] respectively. Readers who have previously read [38] may skip to section Sec.V] In Sec.V], we describe the two general approaches to mapping classical planning problems to QUBO problems, followed by the two problem-type specific mappings. Our methods, particularly the parameters we used for our annealing runs, are described in SecVI SecVII describes and analyzes our results on the scheduling-type family of planning problems, and Sec. VIII describes our few results on the navigationtype family of planning problems. Sec IX examines the embeddability of these problems in future hardware architectures. In Sec $\mathrm{X}$ we conclude with a summary of our results and their implications for the programming and design of quantum annealers.

\section{AN OVERVIEW OF THE CLASSICAL PLANNING FORMALISM}

Classical planning problems, specifically STRIPS planning problems [19, 20, are expressed in terms of binary state variables (sometimes called predicates) and actions (sometimes called operators). Examples of state variables in the rover domain are "Rover $\mathrm{R}$ is in location X" and "Rover R has a soil sample from location X," which may be true or false. Actions consist of two lists,

- a set of preconditions and

- a set of effects (or postconditions).
The set of preconditions can be divided into positive preconditions, those that must be true, and negative preconditions, those that must be false. The set of effects can be divided similarly into a set of positive effects and a set of negative effects. In classical planning, it is conventional that the preconditions for an action must be positive, so the set of preconditions is a subset of state variables that must be set to true in order for the action to be possible to carry out. When this convention is followed, the set of negative preconditions will be empty. The effects of an action consists of a subset of state variables with the values they take on if the action is carried out. For example, the action "Rover $\mathrm{R}$ moves from location $\mathrm{X}$ to location $\mathrm{Y}$ " has one precondition, "Rover $\mathrm{R}$ is in location $\mathrm{X}=$ true" and has two effects "Rover $\mathrm{R}$ is in location $\mathrm{X}$ = false" and "Rover $\mathrm{R}$ is in location $\mathrm{Y}=$ true."

A specific planning problem specifies an initial state, with values specified for all state variables, and a goal, specified values for one or more state variables. As for preconditions, goals are conventionally positive, so the specified value for the goal variables is true. Generally, the goal specifies values for only a small subset of the state variables. A plan is a sequence of actions. A valid plan, or a solution to the planning problem, is a sequence of actions $A_{1}, A_{2}, \ldots, A_{L}$ such that the state at time step $t_{i-1}$ meets the preconditions for action $A_{i}$, the effects of action $A_{i}$ are reflected in the state at time step $t_{i}$, and the state at the end has all of the goal variables set to true.

\section{PARAMETERIZED FAMILIES OF NAVIGATION-STYLE PLANNING PROBLEMS}

Navigation is a critical component in many planning applications and existing planning benchmarks 21, 22, 29. Rover navigation is one such domain. Given a list of locations a rover must visit to, say, take picture or analyze samples, the planner must find a route that makes optimal use of resources, such as time and power, satisfies multiple constraints, and achieves all goals. Under the assumptions that each location need be visited only once, the high-level navigation problem is similar to the Hamiltonian Path problems we investigate.

\section{A. Planning problems from undirected Hamiltonian path (UHP)}

The undirected Hamiltonian path (UHP) problem on an undirected graph $G(V, E)$, with $n$ vertices $V$ and a set of edges $E$, is to find a path that visits each node exactly once. A planning problem instance based on this graph may be formulated as follows. For each vertex $v$, there are:

- An action $a_{v}$ representing visiting $v$. 


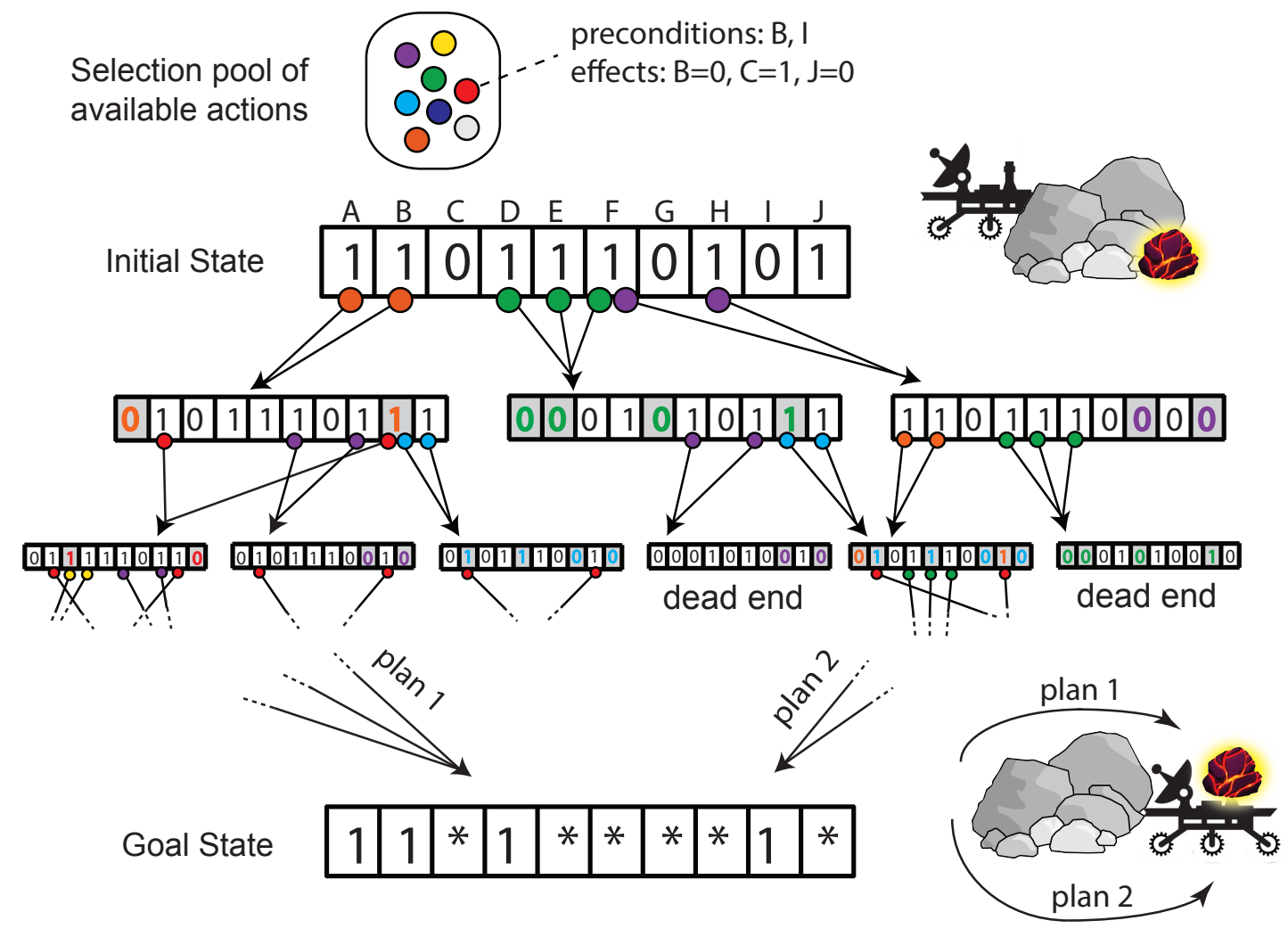

FIG. 1: Pictorial view of a planning problem. The initial state (e.g. Rover to the left behind the rocks, without payload) is specified by assigning True (1) or False (0) to state variables (named A-J in this oversimplified example). The planning software navigates a tree, where a path represents a sequence (with possible repetitions) of actions selected from a pool (colors). Each action has preconditions on the state variables (e.g. moves can be done around the rocks and not through) which need to be satisfied in order for the actions to be executed (the circles under the state variables in the search tree needs to match True $=1$ ) and has an effect on the state (colored variables in shaded regions of the new state have changed values). A valid search plan (multiple valid plans are possible) will reach the goal state (e.g. Rover in front of the rocks to the right, with a sample collected).

- A 'goal' state variable $s_{v}^{g}$ to indicate that $v$ needs to be visited; $s_{v}^{g}=T$ (true) means $v$ has been visited.

- An 'internal' state variable $s_{v}^{i}$ represents whether or not $v$ has been visited. Specifically, $s_{v}^{i}=T$ means $v$ has not been visited while $s_{v}^{i}=F$ (false) means that it has been visited. This variable ensures that each vertex can be visited at most once. While including both $s_{v}^{g}$ and $s_{v}^{i}$ (which always have opposite values) seems redundant, it is necessary because of the convention that allows only positive action preconditions and goals.

- An 'external' state variable $s_{v}^{e}$ represents whether or not the vertex $v$ can currently be visited given the edge structure of the graph. Specifically, it is set to $T$ by an action $a_{v^{\prime}}$ corresponding to visiting a vertex $v^{\prime}$ that is connected to $v$ by an edge. Otherwise, it is set to $F$.
Each action $a_{v}$ has 2 preconditions: (1) $s_{v}^{i}=T$, which indicates that this action has not been used in the plan already, and (2) $s_{v}^{e}=T$, indicating that this action can legally follow the previous action.

Each action $a_{v}$ has $n+1$ effects: (1) $s_{v}^{g}=T$, to indicate that $v$ has been visited, (2) $s_{v}^{i}=F$, thus excluding $a_{v}$ from appearing twice in the plan, (3) sets each of the $n-1$ external variables $s_{v^{\prime}}^{e}$ for each of the other vertices $v^{\prime}$ : if there is an edge from $v$ to $v^{\prime}$ then $s_{v^{\prime}}^{e}=T$, enabling $a_{v^{\prime}}$ to follow $a_{v}$; if there is no edge from $v$ to $v^{\prime}$ then $a_{v}$ sets $s_{v^{\prime}}^{e}=F$, preventing $a_{v^{\prime}}$ from following $a_{v}$.

The initial state has all goal variables $s_{v}^{g}=F$ while all internal and external variables $s_{v}^{i}$ and $s_{v}^{e}$ have value $T$. Thus, any of the $n$ actions $a_{v}$ can be performed at the start. A valid plan is a sequence of the $n$ actions that corresponds to a path along the edges that visits all vertices exactly once.

Problem generation: We obtain a parametrized family of UHP-based planning problems, parametrized by $n$ 
and $p$, by using the Erdös-Rényi model $G_{n, p}$ to randomly generate graphs with $n$ vertices such that, for any pair of vertices, the edge between them is included with probability $p$. We then derive planning problems from these graphs as described in the preceding paragraphs. We use the scaling parameter $p=(\log n+\log \log n) / n$ that has been established as at the phase transition for the closely related Hamiltonian cycle problem [11, 28]. We wrote a simple $\mathrm{C}++$ program to generate these problems.

\section{PARAMETRIZED FAMILIES OF SCHEDULING-TYPE PLANNING PROBLEMS}

Many planning applications include scheduling aspects 12. Scheduling, which deals with assigning resources and time to tasks while taking into account constraints, is in itself an important problem. Certain classes of scheduling problems correspond to graph coloring. For example, a scheduling problem, with a set of tasks and constraints that any pair of tasks competing for the same resource cannot be assigned the same time-slot, can be phrased as a vertex coloring, a well-known NP-complete problem. Specifically, the chromatic number (the smallest number of colors needed) represents the smallest number of time-slots needed to complete a corresponding schedule, thus representing the minimum makespan.

\section{A. Planning problems from Vertex Coloring}

Given an undirected graph $G=\{V, E\}$ with $n$ vertices, the planning problem to color $G$ with $k$ colors is formulated as follows. For each vertex $v$ there are:

- $k$ actions $a_{v}^{c}$ representing coloring $v$ with color $c$.

- A 'goal variable' $s_{v}^{g}$ representing whether or not $v$ has been colored at all.

- A state variable $s_{v}^{c}$ representing whether or not $v$ has been colored with the color $c$.

Let $C(v)$ be the set of neighboring vertices that are connected to $v$ by an edge. For each action $a_{v}^{c}$, there are $|C(v)|+1$ preconditions: (1) $s_{v}^{g}=F$, which indicates that $v$ is not already colored; and (2) for each $v_{i} \in C(v)$, $s_{v_{i}}^{c}=F$, guaranteeing that none of neighboring $v_{i}$ are already colored with color $c$.

Each action $a_{v}^{c}$ has two effects: $s_{v}^{g}=T$ and $s_{v}^{c}=T$.

In the initial state, none of the vertices are colored: $\forall v \in V: s_{v}^{g}=F$, and $s_{v}^{c}=F$. The goal state requires that all vertices are colored: $\forall v \in V: s_{v}^{g}=T$. A plan is a sequence of $n$ actions, each of which colors a vertex $v$.

Problem generation: As for the Hamiltonian path based problems, we obtain a parametrized family of graph-coloring-based planning problems by randomly generating Erdös-Rényi graphs $G_{n, p}$ for a variety of values of $n$ and $p$. A phase transition threshold in the $k$ colorability of $G(n, p)$ graphs has been established for all $k \geq 3$ in terms of the parameter $c=m / n=p \times n$, the ratio of the number of edges to the number of vertices 11. The threshold scales as $c=k \log k$ in the leading term, but the precise location of this threshold is still an open question, even for $k=3$ [14. Our runs were done with $c=4.5$, a value intermediate to the best current lower bound [2] and upper bound [17] for the phase transition. We extended Culberson et al.'s [15] graph generator program, which provides methods to generate different types of graph controlled by various parameters, to output PDDL files containing the specification of planning problems derived from these graphs.

\section{MAPPING PLANNING PROBLEMS TO QUBO FORM}

The D-Wave quantum annealing machine can accept problems phrased in terms of a problem Hamiltonian $H_{P}$ in Ising form:

$$
E_{\text {Ising }}\left(s_{1}, \ldots, s_{N}\right)=-\sum_{i=1}^{N} h_{i}^{\prime} s_{i}+\sum_{\langle i, j\rangle \in \mathrm{E}} J_{i, j}^{\prime} s_{i} s_{j},
$$

where $s_{i}= \pm 1$. In traditional computer science, it is unusual to have variables $s_{i}$ whose values can be taken only from $\{-1,1\}$, but it is common to have binary variables $z_{i}$ that take values from $\{0,1\}$. It is easy to convert between the two forms by taking $s_{i}=1-2 z_{i}$. Any quadratic function of variables $z_{i}$ can be converted to Ising form, up to a constant which does not affect the energy minimization and so can be ignored:

$$
q\left(z_{1}, \ldots, z_{N}\right)=-\sum_{i=1}^{N} h_{i} z_{i}+\sum_{\langle i, j\rangle \in \mathrm{E}} J_{i, j} z_{i} z_{j},
$$

Thus, it suffices to express any problem we want solved as a Quadratic Unconstrained Binary Optimization (QUBO) problem [13, 30, 42, which will then be converted to Ising form to run on the D-Wave machine.

In this section, we describe two different mappings from general classical planning problems, as described in Section III to QUBO form. The first is a time-slice approach. The second approach first maps a planning problem to a constraint satisfaction problem, and then reduces higher order terms to quadratic terms through a series of moves. As we mentioned in Sec. III, some classical planning algorithms follow the convention that an action can have only positive preconditions. Since it is easy to do, we define our mappings generally, so that they work with planning problems in which actions can have negative preconditions, as well as those that follow the convention. 


\section{A. Time-slice method}

This mapping from general classical planning problems to QUBO form is a variant of the one developed and described in 42 .

The method requires setting a specific plan length $L$. For the two families of problems we consider, the plan length $L$ is easy to determine; for the navigation-type problems, it is $n$, the number of sites, and for schedulingtype problems, it is 1 , since all vertices can be colored at the same time. In other cases, it may be necessary to run quantum annealing on QUBOs corresponding to different plan lengths, or to employ more sophisticated techniques to determine the plan length to use $[\underline{6}$.

If the original planning problem has $N$ state variables $x_{i}$ and $M$ actions $y_{j}$ and we are looking for a plan of length $L$, then we define a time-slice QUBO problem in terms of $N(L+1)+L M$ binary variables. There are two groups of binary variables. The first group consists of $N(L+1)$ binary variables $x_{i}^{(t)}$ that indicate whether the state variable $x_{i}$ is 0 or 1 at time step $t$, for $t \in\{0, \ldots, L\}$. The second group consists of $L M$ binary variables $y_{j}^{(t)}$ that indicate whether or not the action $y_{j}$ is carried out between time steps $t-1$ and $t$. We can think of the entire set of binary variables as an alternating string of $N$ variables corresponding to the state at a given time, followed by $M$ variables corresponding to the actions, followed by a $N$ variables corresponding to the state at the next time index, etc. The structure of the QUBO is illustrated in Figure 2.

The total cost function is written as a sum

$$
\begin{aligned}
H= & H_{\text {initial }}+H_{\text {goal }}+H_{\text {no }- \text { op }} \\
& +H_{\text {precond }}+H_{\text {effects }}+H_{\text {conflicts }} .
\end{aligned}
$$

We first give a mapping that is more general than we need, and then explain how it can be simplified in our situation. The first two terms are straightforward. They capture the initial condition and the goal condition. Let $\mathcal{I}^{(+)}$be the set of state variables that are 1 in the initial condition and $\mathcal{I}^{(-)}$be the set of state variables that are initially set to 0 . Similarly, let $\mathcal{G}^{(+)}$be the set of goal variables with value 1 and $\mathcal{G}^{(-)}$be the set of goal variables with value 0 . (We describe the mapping for general classical planning problems that do not necessarily follow the convention that preconditions and goals must be positive.) To capture the boundary conditions, the requirement that a plan start in the appropriate initial state and meets the goals, we include the following terms in the cost function:

$$
H_{\text {initial }}=\sum_{i \in \mathcal{I}^{(+)}}\left(1-x_{i}^{(0)}\right)+\sum_{i \in \mathcal{I}^{(-)}} x_{i}^{(0)}
$$

and

$$
H_{\text {goal }}=\sum_{i \in \mathcal{G}^{(+)}}\left(1-x_{i}^{(L)}\right)+\sum_{i \in \mathcal{G}^{(-)}} x_{i}^{(L)}
$$

$\mathrm{N}$ state variables

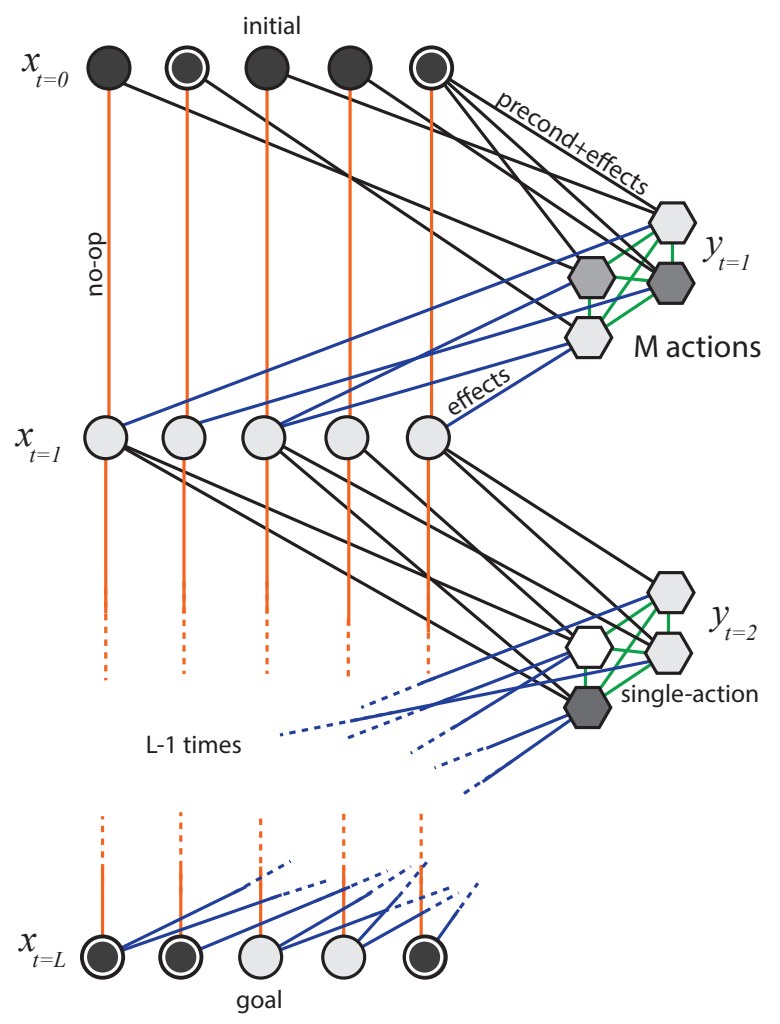

FIG. 2: Time-slice QUBO structure for a planning problem with only positive preconditions and goals. Each node represents a state variable (left) or an action (right) at any given time $t$. Time flows from top to bottom, and variables $y_{i}^{(t)}$ for the actions at time $t$ are shown between the state variables $x_{i}^{(t-1)}$ for one time step and the state variables $x_{i}^{(t)}$ for the next time step. The node grayscale intensity represents the magnitude of local field (bias) $h_{i}$ applied to a given qubit $i$, and the double contour in a node indicates a negative bias. (One interesting property of this mapping is that the nodes representing state variables for $t \neq 0$ and $t \neq L$ have the same $h$ value, since they all come from $H_{n o-o p}$. For this reason, they are all shown in same color in the diagram). Edges represents the couplings $J_{i j}$. Their weight is not illustrated in the figure. In this example we consider $H_{\text {single-action }}$ instead of $H_{\text {conflicts }}$, so all of the actions at a given time step are coupled to each other.

We next add terms to the cost function that penalize a plan if an action is placed at time $t$ but the prior state does not have the appropriate preconditions or if the subsequent state does not reflect the effects of that action. Furthermore, we must penalize variable changes that are not the result of an action. We start with this term, the $H_{\text {no-op }}$ term, that penalizes variable changes:

$$
H_{\mathrm{no}-\mathrm{op}}=\sum_{t=1}^{L} \sum_{i=1}^{N}\left[x_{i}^{(t-1)}+x_{i}^{(t)}-2 x_{i}^{(t-1)} x_{i}^{(t)}\right]
$$

This term gives cost penalty of 1 every time a variable is flipped. Of course, when the effect of an action does 
result in a variable flipping, we do not want this penalty, so we will make up for this penalty when we add the term that corresponds to the effects of an action. First, the term that penalizes violation of the preconditions looks like

$$
\begin{aligned}
H_{\text {precond }}= & \sum_{t=1}^{L} \sum_{j=1}^{M}\left(\sum_{i \in \mathcal{C}_{j}^{(+)}}\left(1-x_{i}^{(t-1)}\right) y_{j}^{(t)}\right. \\
& \left.+\sum_{i \in C_{j}^{(-)}} x_{i}^{(t-1)} y_{j}^{(t)}\right)
\end{aligned}
$$

where $\mathcal{C}_{j}^{(+)}$is the set of positive preconditions for action $j$ and $\mathcal{C}_{j}^{(-)}$is the set of negative preconditions. Let $\mathcal{E}_{j}^{(+)}$be the set of positive effects for action $j$ and $\mathcal{E}_{j}^{(-)}$the set of negative effects. The penalty if the appropriate effects do not follow the actions is captured by the following term:

$$
\begin{aligned}
H_{\text {effects }}= & \sum_{t=1}^{L} \sum_{j=1}^{M}\left(\sum_{i \in \mathcal{E}_{j}^{(+)}} y_{j}^{(t)}\left(1+x_{i}^{(t-1)}-2 x_{i}^{(t)}\right)\right. \\
& \left.+\sum_{i \in \mathcal{E}_{j}^{(-)}} y_{j}^{(t)}\left(2 x_{i}^{(t)}-x_{i}^{(t-1)}\right)\right)
\end{aligned}
$$

In order to understand this term, we must consider it together with the no-op term. When $y_{j}^{(t)}=1$, the corresponding term for $i \in \mathcal{E}_{j}^{(+)}$(resp. $i \in \mathcal{E}_{j}^{(-)}$), taken together with the no-op term, can be written

$$
\left(1+2 x_{i}^{(t-1)}\right)\left(1-x_{i}^{(t)}\right)
$$

(resp.

$$
\left(3-2 x_{i}^{(t-1)}\right) x_{i}^{(t)}
$$

for negative effects), resulting in a positive penalty unless $x_{i}^{(t)}=1$ (resp. $x_{i}^{(t)}=0$ ). By using this form we have corrected for the corresponding no-op term.

Classical planners often allow for parallel plans not just "linear plans" in which more than one action can take place at one time if those actions could have been done in any order, meaning that the effects of any one action do not conflict with preconditions of the other actions. What we have done so far works fine when the preconditions mean that only one action can take place per time period as is the case in the navigation problems. In the scheduling problems, multiple actions can take place at the same time without conflicting. For general planning problems, we can either rule out multiple actions by imposing an additional term

$$
H_{\text {single-action }}=\sum_{t=1}^{L}\left(\sum_{j=1}^{M} y_{j}^{(t)}-1\right)^{2},
$$

or we need to add terms to penalize potential conflicts. To complicate matters, when more than one action can take place at a given time, we are in danger of overcorrecting for the no-op term. If multiple actions at the same time have the same effect, the $H_{\text {effects }}$ term will add a term for each of those actions, thus overcompensating for the no-op penalty. To avoid overcompensating, we penalize multiple actions at the same time having the same effect, discouraging all such actions. A less stringent way to avoid overcompensating would be to add this penalty only when the effect changes the variable, as we have done in the no-op term. The problem is that natively that is not a quadratic term. Of course one could then reduce that term, but here we choose to use the more stringent solution. To ensure that two actions that conflict in the sense that positive preconditions of one overlap with negative effects of the other or vice versa, and to avoid overcompensating, we include the penalty

$$
\begin{aligned}
H_{\text {conflict }}= & \sum_{t=1}^{L} \sum_{i=1}^{N}\left(\sum_{j \mid i \in \mathcal{C}_{j}^{(+)} \cup \mathcal{E}_{j}^{(-)}} \sum_{j^{\prime} \neq j \mid i \in \mathcal{E}_{j^{\prime}}^{(-)}} y_{j}^{(t)} y_{j^{\prime}}^{(t)}\right. \\
& \left.+\sum_{j \mid i \in \mathcal{C}_{j}^{(-)} \cup \mathcal{E}_{j}^{(+)}} \sum_{j^{\prime} \neq j \mid i \in \mathcal{E}_{j^{\prime}}^{(+)}} y_{j}^{(t)} y_{j^{\prime}}^{(t)}\right) .
\end{aligned}
$$

While for explanatory purposes it was useful to include variables for the state at time $t=0$, those can be set ahead of time, so that we don't need to include the $H_{\text {initial }}$ term. The same is true of the $H_{\text {goal }}$ term. We can also replace all of their occurrences in $H_{\text {no-op }}, H_{\text {condition, }}$, and $H_{\text {effect }}$ with these set values to simplify those constraints. Furthermore, if a state variable first appears at time step $t$, then in the no-op term connecting it with the previous level, we can set it to 0 . Finally, for the final action time slot, we can remove any actions whose effects conflict with the goals or do not contribute to the goals. These simplifications result in modified terms $H_{\text {no-op }}^{\prime}, H_{\text {condition }}^{\prime}$, and $H_{\text {effect }}^{\prime}$. Additionally, since in our setting we have followed the convention that preconditions must be positive, we can use a simpler version of the $H_{\text {precond }}$ term:

$$
H_{\text {precond }}^{\prime}=\sum_{t=1}^{L} \sum_{j=1}^{M} \sum_{i \in \mathcal{C}_{j}^{(+)}}\left(1-x_{i}^{(t-1)}\right) y_{j}^{(t)}
$$

For the navigation problems, the QUBO simplifies to

$$
H=H_{\mathrm{no}-\mathrm{op}}^{\prime}+H_{\text {precond }}^{\prime}+H_{\mathrm{effects}}^{\prime}
$$

and for the scheduling problems the QUBO simplifies to

$$
\begin{aligned}
H= & H_{\mathrm{no}-\mathrm{op}} \\
& +H_{\text {precond }}^{\prime}+H_{\text {effects }}+H_{\text {single-action }} .
\end{aligned}
$$




\section{B. CNF approach}

A CNF expression over $n$ Boolean variables $x_{i}$ consists of a bunch of clauses $C_{a}$ consisting of $k$ variables, possibly negated, connected by logical ors:

$$
b_{1} \vee b_{2} \vee \cdots \vee b_{k}
$$

where

$$
b_{i} \in\left\{x_{1}, x_{2}, \ldots, x_{n}, \neg x_{1}, \neg x_{2}, \ldots, \neg x_{n}\right\},
$$

and the number of variables $k$ in the clause can vary from clause to clause. A CNF for a $k$-SAT expression consists of clauses that all have the same number of variables $k$. In a CNF coming from 2-SAT, for instance, all clauses have the form $b_{1} \vee b_{2}$. In a CNF, all of the clauses must be satisfied, which means they are connected by an AND operator (the reason for the "conjunctive" in "conjunctive normal form").

We used the first of the four PDDL to CNF translators built in to the SATPLAN planner [26, a classical SATbased planner, to output planning problems in conjunctive normal form (CNF). Compilation planners such as SATPLAN perform some preprocessing, such as "reachability" and "relevance" analysis, as part of the translation to reduce the size of the output. Reachability analysis makes a quick determination as to whether a given action can appear at a given time step, and removes from consideration at that time step any actions that it has determined cannot be carried out, which results in a simplified CNF expression. Similarly, if the possible actions at previous time steps cannot change a state variable, the resulting expression is simplified accordingly. For example, in the first time step, only actions whose preconditions are satisfied in the initial state are considered. Then, only values of state variables that occurred in the initial state or are effects of actions considered at the first time step are considered. This process is iterated until it no longer results in simpification. Relevance analysis is a similar process that starts its analysis in the last time step, considering only state variables that are goals, and then working backwards leaving only actions with effects relevant to these goals in the last action time step. Relevant state variables at time step $t$ represent the union of state variables at time step $t+1$ and the preconditions of all relevant actions at time step $t$. SATPLAN's "actionbased" encoding uses an advanced reachability and relevance heuristic analysis, and then further removes all variables representing state variables while adding constraints that capture the relationships between actions in consecutive time steps that were previously enforced by relationships between actions and state variables.

We convert a CNF instance to QUBO by first transforming it to Polynomial Unconstrained Binary Optimization (PUBO), a generalization of QUBO in which the objective function is a pseudo-Boolean of arbitrary degree. For each clause in a given CNF instance, we introduce a term to the PUBO instance equal to the conjunction of the negation of the literals in that clause, where a positive literal is replaced by the corresponding binary variable and a negative literal is replaced by the difference of one and the corresponding binary variable. For example, the CNF term $\left(x_{1} \vee \neg x_{2} \vee \neg x_{3} \vee x_{4}\right)$ would correspond to the PUBO term $\left(1-x_{1}\right) x_{2} x_{3}\left(1-x_{4}\right)$.

We then reduce higher degree terms in the PUBO instance using an iterative greedy algorithm that is related to one described in 9. At each step, the pair of variables that appears in the most terms is replaced by an ancilla variable corresponding to their conjunction. (If there are multiple such pairs, then one is chosen arbitrarily.) A penalty term is introduced to enforce that the ancilla variable indeed corresponds to the requisite conjunction. The penalty weight we use is equal to one plus the greater of the sums of the magnitudes of the positive coefficients and negative coefficients of the terms the ancilla is used to reduce [4. The one is added to ensure that the constraint-satisfying states have lower energy than the constraint-violating states. One is convenient, and in keeping with the integer coefficients for the other terms, but any positive constant would do. This procedure is repeated until the resulting PUBO is quadratic.

\section{Direct mapping of underlying graph problems to QUBO}

We now turn to two more compact, but problemtype specific mappings, the first mapping navigation-type problems to QUBO problems and the second mapping scheduling-type problems to QUBO.

\section{Direct mapping of graph coloring to QUBO}

For a graph coloring problem with $n$ vertices and $k$ colors, we have $k n$ binary variables, $x_{i c}$, where $x_{i c}=1$ means that vertex $i$ is colored with color $c$, and $x_{i c}=0$ means it is not.

The QUBO contains two different types of penalty terms. The first corresponds to the constraint that each vertex must be colored by one, and no more than one, color: $\sum_{c=1}^{k} x_{i c}=1$. So for each vertex $i$, we have a term

$$
\left(1-\sum_{c=1}^{k} x_{i c}\right)^{2} \text {. }
$$

The second corresponds to the constraint that two vertices connected by an edge cannot be colored with the same color. For each vertex $i$, we have a term

$$
\sum_{(i, j) \in E} \sum_{c} x_{i c} x_{j c} .
$$

Altogether the QUBO is

$$
\sum_{i=1}^{n}\left(1-\sum_{c=1}^{k} x_{i c}\right)^{2}+\sum_{(i, j) \in E} \sum_{c} x_{i c} x_{j c} .
$$




\section{Direct mapping of Hamiltonian Path Problems to QUBO}

For a Hamiltonian path problem with $n$ sites, we have $n^{2}$ variables

$$
\left\{x_{11}, \ldots, x_{1 n}, x_{21}, \ldots, x_{2 n}, \ldots, x_{n 1}, \ldots, x_{n n} \cdot\right\}
$$

The first index $i$ gives the site, the second index gives the time slot, so $x_{i j}=1$ means that the $i$ th site is the $j$ th site visited, and $x_{i j}=0$ means that the $i$ th site is not visited in the $j$ th time slot.

There are three types of terms in the QUBO cost function: penalties if a site is visited more or less than once, penalities if more than one site is visited in a given time slot, and penalities for violation of edge constraints.

The first type of term enforceses that each site is visited exactly once: For each site $i$, we will have a term of the form

$$
\left(\sum_{j} x_{i j}-1\right)^{2}
$$

The second type of term enforces that in each time slot no more than one site is visited (we may as well enforce it to be exactly one): For each time slot $j$, we have a term of the form

$$
\left(\sum_{i} x_{i j}-1\right)^{2}
$$

The third type of term is a single term penalizing the violation of edge constraints. It penalizes bisiting the $i^{\prime}$ th site right after the $i$ th site if they are not connected by an edge:

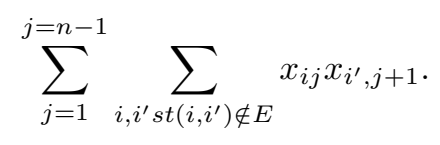

There are $2 n+1$ terms all together.

\section{METHODS}

All quantum annealing runs were performed on the 509-qubit D-Wave Two machine housed at NASA Ames. In all cases, we used an annealing time of $20 \mu \mathrm{sec}$, the fastest annealing time currently available on the machine, which is also D-Wave's recommended annealing time given that current data suggests that an even faster annealing time would be optimal 39. For each embedded QUBO instance, we performed 45,000 anneals at each of ten gauges, for a total of 450,000 anneals per QUBO instance. Gauges, which determine whether the bit values $\{0,1\}$ of each QUBO variable are mapped to $\{-1,1\}$ or $\{1,-1\}$, are used to reduce the effects of asymmetries in the hardware 35, 39.

From a QUBO instance generated as described in Section $\mathrm{V}$, we generate the embedded instance by running DWave's heuristic embedding software [10] on the original
QUBO instance. We use the software's default parameters, including a maximum of 10 tries, unless otherwise noted. The output of the embedding software is a set of disjoint connected components of the hardware graph $C_{i}$, one for each variable $x_{i}$ in the original QUBO. We performed our own parameter setting, rather than using D-Wave's which tries successive parameter values, so that we could obtain statistics for a variety of parameter settings.

From the original QUBO instance, we obtain the logical Ising instance

$$
h_{i} s_{i}+J_{i j} s_{i} s_{j}
$$

through the standard translation of the variables $s_{i}=$ $1-2 z_{i}$. To obtain the embedded Ising instance, we evenly distribute the bias $h_{i}$ in the logical Ising instance among the qubits corresponding to the nodes in $C_{i}$ : we set the linear coefficient for each variable $y$ in $C_{i}$ to be $\frac{h_{i}}{\left|C_{i}\right|}$. We set all internal couplings, couplings between physical qubits that represent the same logical variable in the original QUBO, to the internal coupling constant $J_{\text {int }}$, a value we specify; the coefficient of all quadratic terms $y y^{\prime}$ such that $y$ and $y^{\prime}$ are both in $C_{i}$ for some $i$ are set to $J_{\text {int }}$. We describe shortly the results we obtained in experiments varying this value. The only other couplings are between sets of physical qubits $C_{i}$ and $C_{j}$ representing two different logical variables $x_{i}$ and $x_{j}$ that appear together in a quadratic term in the original QUBO. In many cases there is only one edge in the hardware graph between the qubits in $C_{i}$ and $C_{j}$. In this case, we set the coupling between them to $J_{i, j}$. When more than one edge exists between these sets, we choose one of them, and set its coupling to $J_{i, j}$. All other couplings are set to zero.

Our test set consists of 100 solvable problem instances at the phase transition for each size for both planning problem types. We generated the problems as described in 38, and then took the first 100 solvable problems. For the sizes in which 38] already had generated problems, we use the first 100 solvable instances tested there. The smallest size problems we considered of the navigationtype were of size 4 . For the scheduling-type problems, we started at size 8 because the phase transition parameter is inaccurate for smaller sizes, biasing the results toward unsolvable instances.

Because all of the problems we consider are solvable, we know the ground state energy in all cases; zero, the minimal value of the QUBO in all cases is attainable, and from that we can compute the ground state energy of the embedded Ising problem that was actually run. For each embedded instance, once we obtain the 450,000 results from the run, we check how many times the ground state energy was obtained, which gives us the probability of solution $r$ for a $20 \mu$ sec anneal. We then compute the expected number of runs $k=\frac{\ln (1-0.99)}{\ln (1-r)}$ required to obtain a $99 \%$ success probability, multiply by the anneal time of $20 \mu \mathrm{sec}$, and report $20 k \mu \mathrm{sec}$, the expected total anneal time to obtain a $99 \%$ success probability. We 
are effectively using a $0.9 \mathrm{sec}$. cutoff time, since the expected anneal time when only one anneal solves is 0.9 secs. Given that classical planners solve these problems in less than 0.1 secs., with the best planners for these problems solving them in less than 0.01 secs. [38], this cutoff time seems reasonable.

We report the median expected total anneal time across 100 instances, with error bars corresponding to the 35 th and 65 th percentiles. Thus each data point shown represents 45 million anneals. While the total annealing time for each point is only 90 seconds, because the read-in and read-out take considerably longer than the anneal time, and because of contention for the machine, the wall clock time to obtain a single data point is hours not minutes. Finding the embedding, by far the longest step in the process, can take minutes for the largest instances, but fortunately needs to be performed only once per QUBO instance.

The ground state energy of the embedded Ising model will not be obtained if the final bit values differ between any two physical qubits representing the same logical qubit. This observation suggests a simple, totally classical error correction scheme that uses majority voting among all physical qubits representing the same logical qubit. This error correction scheme is working at a different level from that in Pudenz et al. [36]; the benchmark problems in Pudenz et al. are native problems that do not require an embedding step in order to be run on the hardware. We report expected total anneal time both with and without this simple form of error correction.

\section{RESULTS ON SCHEDULING-TYPE PLANNING PROBLEMS}

Because the scheduling-type planning problems embedded much more easily than the navigation-type planning problems, we were able to do significantly more analysis of the the choices affecting the D-Wave Two's performance on these problems than on the navigationtype problems. We first examine performance of the DWave Two on scheduling-type instances mapped using the two general approaches for mapping planning problems to QUBOs. We then turn to the results on these instances using the mapping specific to the scheduling approach, the direct mapping from scheduling-type planning problems to QUBO.

\section{A. Time-slice and CNF mapping results}

Fig. 3 shows the relative performance, in terms of median expected total annealing time for $99 \%$ percent success, of the D-Wave Two on the benchmark set of scheduling-type planning problems. When at least half of the instances do not solve within the 0.9 sec. effective cutoff time, we no longer show the point. For the CNF mapping, that happens already by problem size 11. For

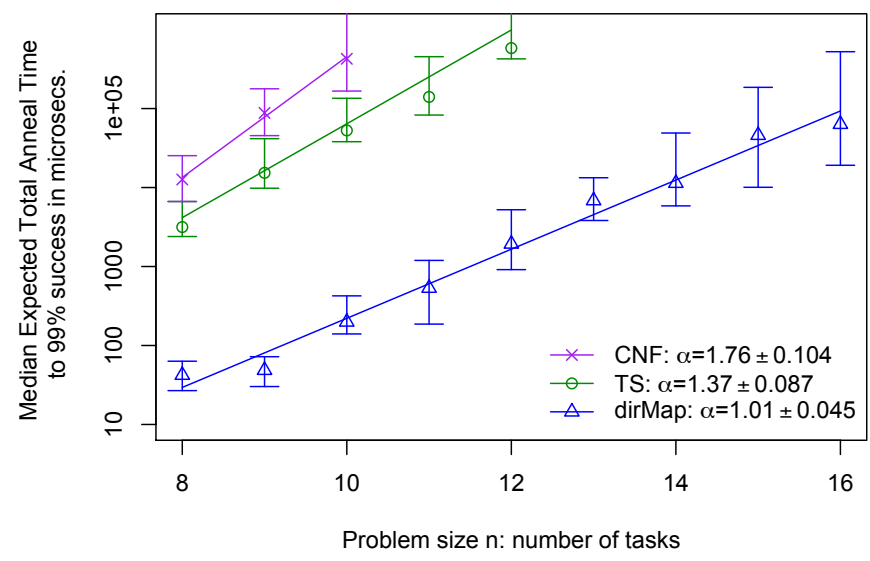

FIG. 3: Comparison of the median expected total anneal time to $99 \%$ percent success for the three mappings of scheduling-type planning problems. The results with the best $J_{\text {int }}$ are shown. (See Sec. VIID and Fig. 10 for how these values were determined.) For the CNF mapping, $J_{\text {int }}$ was $\{1.2,1.3,1.4\}$ for problem sizes $\{8,9,10\}$. For the time-slice mapping, $J_{\text {int }}$ was $\{1.6,1.7,1.7,1.7,1.6\}$ for problem sizes $\{8,9,10,11,12\}$. For the direct mapping, $J_{\text {int }}$ was $\{1.1,1.1,1.3,1.3,1.3,1.4,1.4,1.4,1.4\}$ for problem sizes $\{8, \ldots, 16\}$. Each data point shows the median expected total annealing time to achieve $99 \%$ success over the 100 problems of each size given on the $\mathrm{x}$-axis. The error bars are at the 35 th and 65 th percentiles. When at least half of the instances do not solve within the $0.9 \mathrm{sec}$. effective cutoff time, we no longer show the point. Also, when fewer than $65 \%$ solve, the top of the error bar is indeterminate, as happened for the last point shown in both the CNF and time-slice series.

the time-slice instances, at least half do not solve within the cutoff time by problem size 13, and for the direct map by problem size 17 .

Not surprisingly, the median expected total anneal time to $99 \%$ success is substantially lower for the direct map instances than for the time-slice or CNF instances; it is about a factor of 100 smaller for all problem sizes in the range. The direct mapping is tailored to this particular kind of scheduling-type planning problems, rather than being applicable to planning problems in general. For this reason, the QUBO mapping is much more efficient (Fig. 4), exactly $3 / 8$ smaller than the time-slice QUBOs across the entire size range. The typicial embedding size (Fig. 6) is also smaller, roughly half that of the other mappings. For these reasons, it is to be expected that the performance on the directly mapped problem instances is significantly better than the performance for either of the general-purpose mappings that, unlike the direct mapping, can be applied to any planning problem.

More surprising is the substantial difference between the performance on the time-slice mapped instances and the CNF mapped instances, with the median expected total annealing time to achieve $99 \%$ success being about a factor of 5 greater for the CNF instances than the time-slice instances (Fig. 3). The scaling for the timeslice approach is also significantly better than for the 


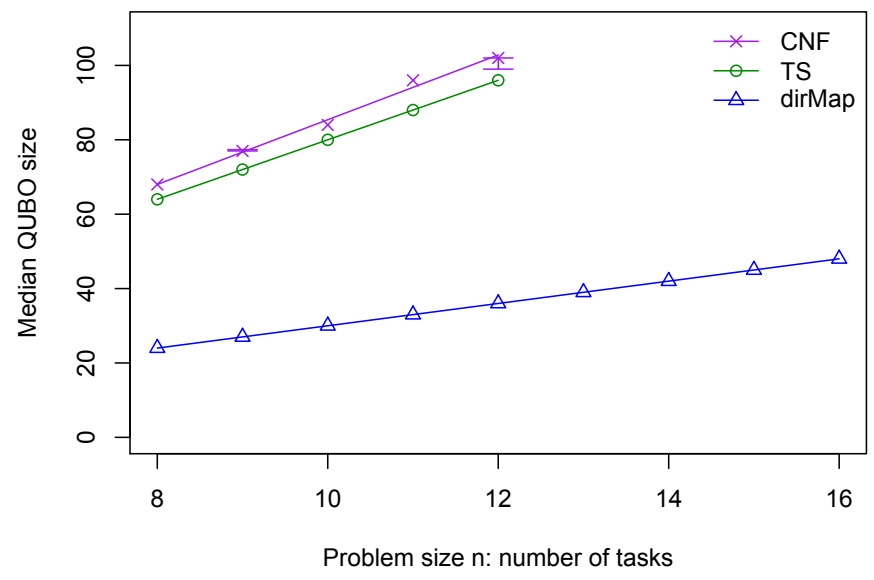

FIG. 4: Comparison of QUBO size across mappings of the scheduling-type planning problems. The QUBO size for the direct map is simply $3 n$, and for the time-slice map it is $8 n$, where $n$ is the number of tasks that need to be scheduled. The QUBO sizes for the CNF mapping vary, so for this case, we are showing the median QUBO size over the 100 problems of that size. Some of the error bars for the CNF mapping at the 35 th and 65 th percentiles are too small to see.

CNF approach, with an $\alpha$ value of 1.37 rather than 1.76 (though the scaling is estimated on very few data points). The time-slice and CNF mappings yield comparablysized QUBOs (Fig. 4), with similar numbers of computings (Fig. 5). For problem size $n$, the time-slice mapping yields a QUBO of size $8 n$ qubits. The CNF approach yields variable size QUBOs, with the median size CNF QUBO over 100 problems only 4 to 8 qubits larger than the median size of the time-slice QUBOs for problem sizes $\{8,9,10,11,12\}$. The median number of couplings for the CNF QUBOs exceeds that of the time-slice QUBOs by only $\{8,10,8,16,9\}$ for problem sizes $\{8,9,10,11,12\}$ respectively. Even the median embedding sizes of the CNF QUBOs are only $\{7,14,26,28,12\}$ qubits larger respectively than the embedded time-slice QUBOs in this range, no more than a $10 \%$ difference across this range (Fig. 6).

\section{B. Comparing embedding properties across mappings}

While the difference in performance between the CNF and time-slice instances is likely due in part to the somewhat larger size of the embedded CNF QUBOs, other factors likely contribute to this difference. One possible factor is the size of the embedding components, the number of physical qubits representing a logical qubit. For example, D-Wave recommends both minimizing the size of the embedding and the maximum component size in an embedding [10]. Also, for anti-ferromagnetic chains, Pudenz et al. 36. showed that the performance of the D-Wave Two decreases with chain size. Because in our

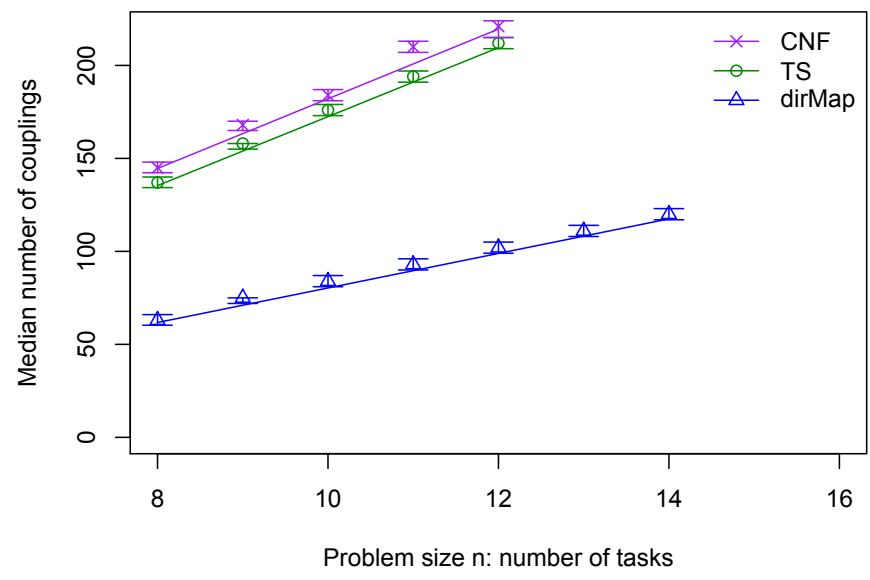

FIG. 5: Comparison of the number of couplings in the QUBOs obtained from the three different mappings of the scheduling-type planning problems. The median number of couplings in the QUBOs for the 100 problems of each size is shown, with error bars at the 35th and 65th percentiles.

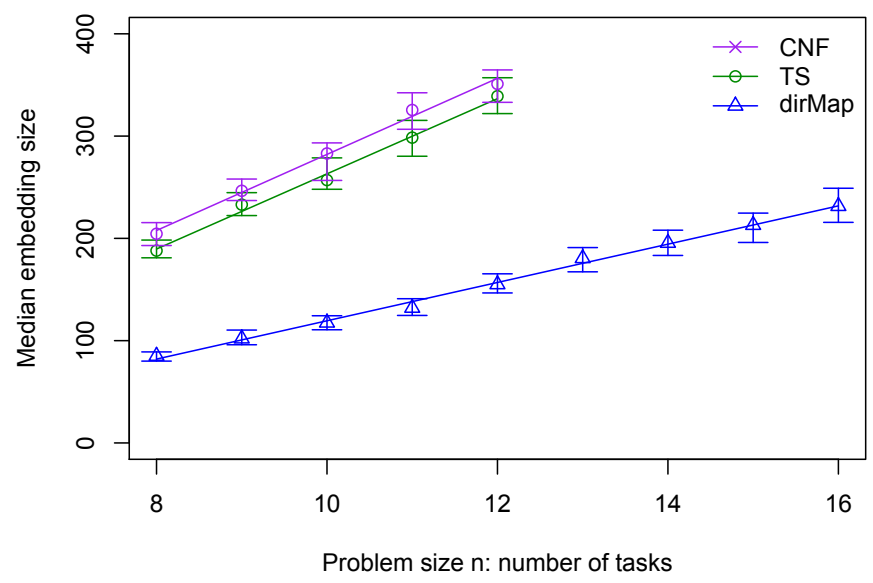

FIG. 6: Comparison of embedding size across mappings of the scheduling-type planning problems. Median embedding size over the 100 problems of size $n$. The error bars are at the 35 th and 65 th percentiles.

case the components can have a more complex topology than a single chain, are subject to local fields, and have couplings to qubits outside the component, it is unclear to what extent we would see the same behavior. Thus, the relative component sizes require investigation.

Fig. 7 (Top) confirms, as would be expected given the similar enbedding and QUBO sizes for the CNF and timeslice instances, that the median average component size across the 100 problems is statistically indistiguishable in the two cases across the range of sizes. Furthermore, throughout the size range tested, the median median component size - the median over the 100 problem instances of the median component size of each instance - and even the median 65 th percentile component size, for both mappings is 1 . By the 90th percentile, the component size has increased significantly in both cases, but 

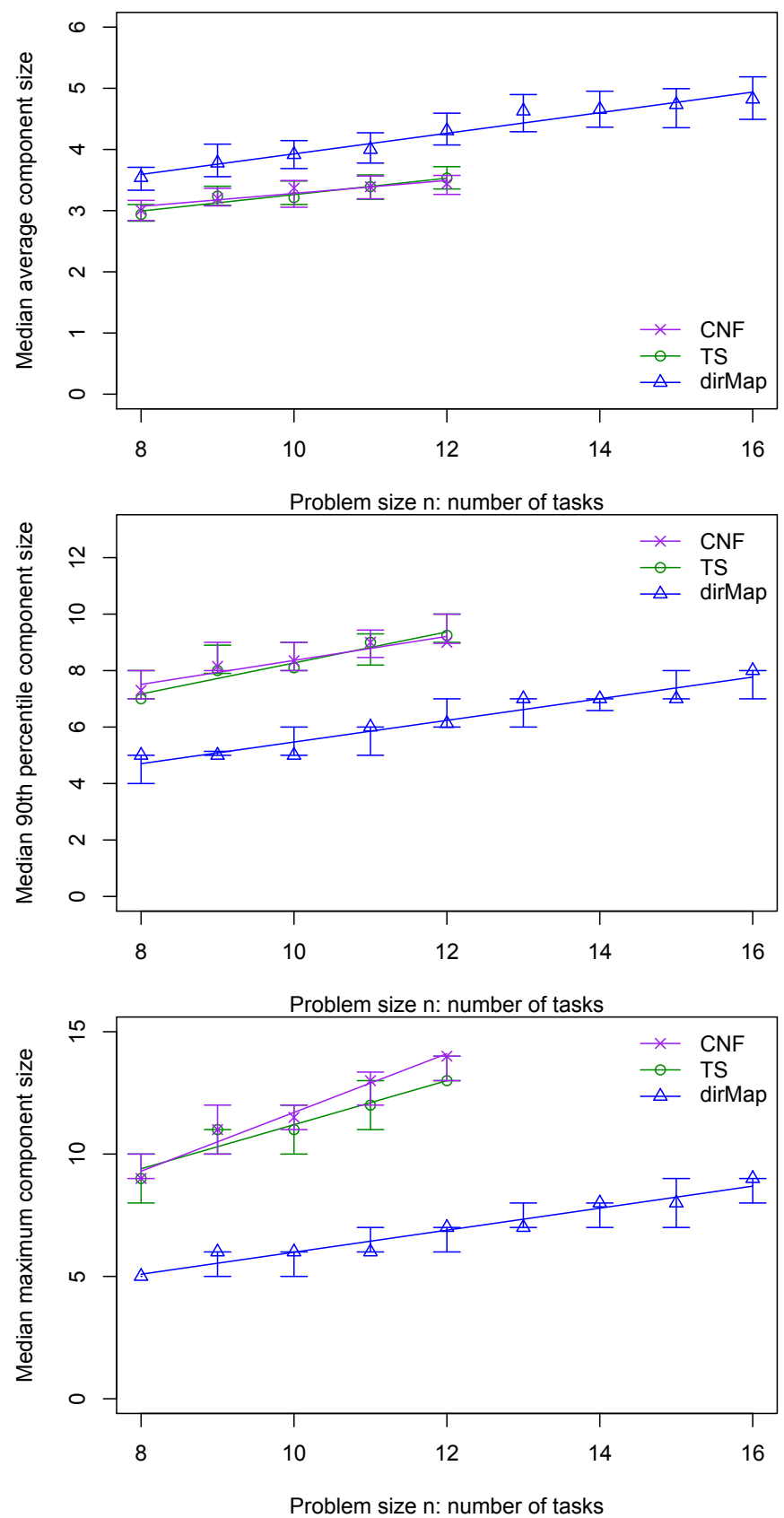

FIG. 7: Comparison of component sizes for schedulingtype planning problems. Median average component size (Top): We computed the average of the component sizes, the number of physical qubits representing each logical qubit, and then took the median over all the 100 problem instances of that size. Median 90th percentile component size (Middle): We calculated the component size at the 90th percentile for each problem, and then took the median over all the 100 problem instances of that size. Median maximum component size (Bottom): We determined the largest component size for each problem, and then took the median over all 100 instances of each size. Error bars are at the 35 th and 65 th percentiles. the two cases are statistically indistinguishable (Fig. 7 (Middle)). Even the median maximum component size hardly differs between the two mappings (Fig. 7] (Bottom)): for problem sizes $n=\{8,9,10,11,12\}$, the median maximum component size for the time-slice instances was $\{9,11,11,12,13\}$, whereas for the CNF instances it was $\{9,11,11.5,13,14\}$. Given that the median maximum component size was identical for the two smallest sizes, even though the performance was markedly different in the two cases, and the difference between the median maximum component sizes was no more than 1 for the other sizes, we conclude that component size did not contribute significantly to the difference in performance between the time-slice and CNF instances.

Even though the QUBO size under the direct map is never much more than roughly half the size of the QUBOs under the two general mappings in the size range considered, both in terms of number of qubits (Fig. 4) and the number of couplings (Fig. 5), the typical component size in the embedded direct mapping instances is markedly larger than for the CNF and time-slice mappings. Fig. 7 shows the median average component size. The direct map typical component size is significantly greater than the typical component size for the $\mathrm{CNF}$ and time-slice mappings throughout the range of problem sizes. Even at problem size 8 it is markedly higher than for the other two. While, the median median component size was 1 for both the CNF and time-slice mappings throughout the range considered, the median median component size for the direct map started at around 4 at size 8 and rose to close to 4.5 by problem size 18 .

The size of the top 10 percent of the components in these embeddings, however, is markedly lower than that of the CNF and time-slice mappings; even at problem size 16 it is still lower than the value for problem size 8 for the more general mappings. These findings are consistent with D-Wave's recommendation to minimize the maximum component size, not just the total embedding size (or equivalently, the typical component size). The results for the time-slice versus CNF instances of the same underlying scheduling-type planning problems suggest that further investigation is needed as to what properties of mappings and embeddings correspond to better or worse performance, and that more sophisticated metrics for good embeddings are needed.

\section{Comparing two different annealing profiles}

The D-Wave Two machine has fixed functions $A(s)$ and $B(s)$ for the weights of the driver and problem Hamiltonians in the control Hamiltonian of Eqn. 1. The user can vary only the overall annealing time, and as mentioned, the overwhelming evidence is that for the present machine the shortest possible annealing time is best. Inadvertently, we were able to experiment with an alternate annealing profile, albeit a nonstandard one. The D-Wave Two has two annealing lines, both common to 


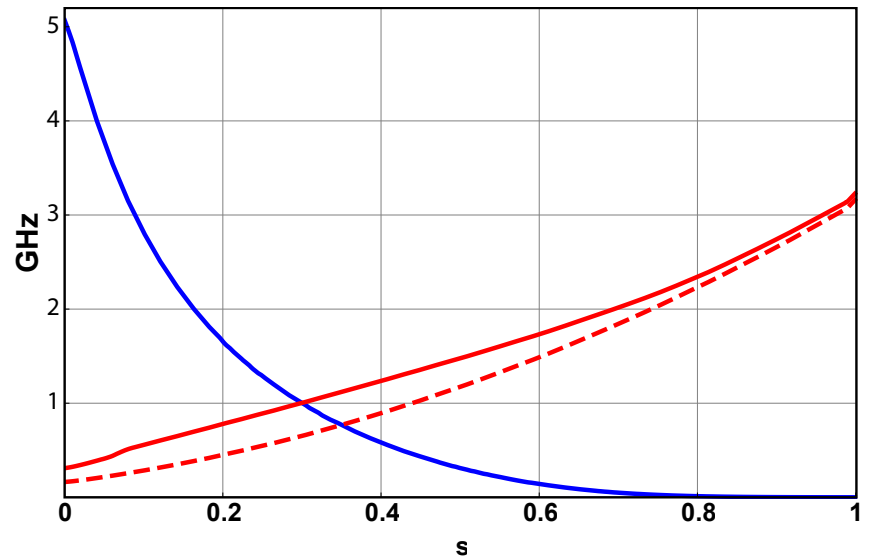

FIG. 8: Weightings of the different components of the Hamiltonian prior to recalibration. The figure shows the discrepancy between the strength $B^{\prime}(s)$ of the local fields (dashed line) and the strength $B(s)$ of the couplings (solid line) in the problem Hamiltonian during annealing prior to recalibration as they increase throughout the anneal. The strength of the driver Hamiltonian as it decreases throughout the anneal is also shown. Normally in an anneal, there is only a single weighting for the problem Hamiltonian, as in Eqn 1 Indeed, after recalibration, the strength of the local fields has been adjusted to equal that of the couplings, so $B^{\prime}(s)$ becomes $B(s)$.

all qubits. These lines affect the local fields $h_{i}$ and coupling coefficients $J_{i j}$ differently. When they are not fully synchronized, effectively the $B(s)$ weighting splits into two weightings, the original $B(s)$ weighting for the couplings and a $B^{\prime}(s)$ weighting for the local fields. When we first ran, these two lines were not fully synchronized. While the two converged near the end of the anneal, for much of the time, the strength of the local fields for the problem Hamiltonian was $2-3 \mathrm{GHz}$ less than that of the coupling strength for the problem Hamiltonian (Fig. 8). The synchronization issue was fixed by estimating the time-dependence of the persistent current and modifying the signal in annealing lines so as to compensate for the effect and make the weights on the coupling and local fields uniform.

Fig. 9 shows results on directly mapped instances both before and after the recalibration. While the recalibration improved results for problems other groups were running on the machine, it resulted in a substantial decrease in performance on the directly mapped scheduling-type planning problems, both in terms of the absolute total annealing time and in the scaling, with $\alpha$ increasing from 0.6 to 1.0 .

\section{Performance dependence on the internal coupling}

We now turn to how the value of $J_{\text {int }}$ affects performance for each of the three different mappings. Naively,

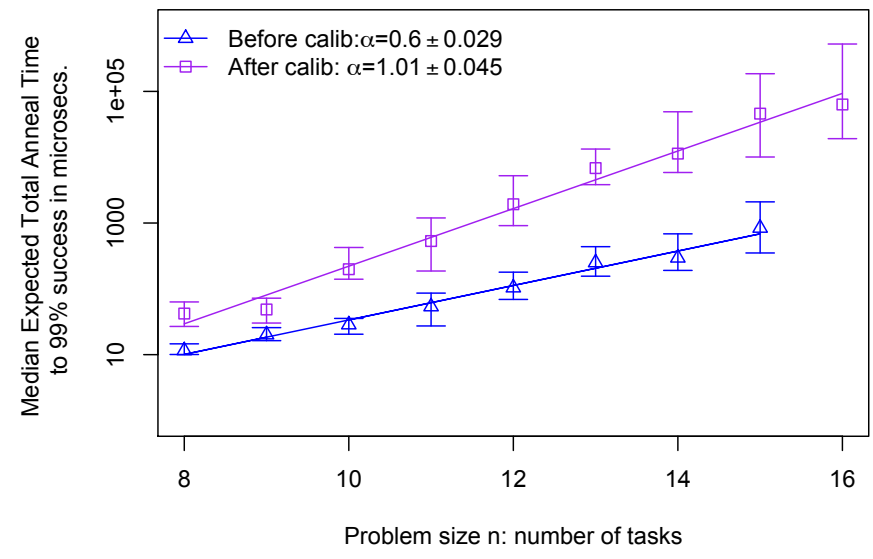

FIG. 9: Median expected total anneal time before and after a recalibration. The performance on the directly mapped scheduling-type planning problems decreased significantly after recalibration. Shown is the median expected total anneal time for $99 \%$ percent success for $J_{i n t}=1.25$ before the recalibration, and the results for the best $J_{\text {int }}$ after calibration (same as in Fig. 3). The variability in the expected total anneal time across the 100 problems also increased, as illustrated by the error bars at the 35 th and 65 th percentiles. While the recalibration improved results for other problems run on the machine, the reverse held for the planning problems we ran.

one might expect that one should set $J_{\text {int }}$ as high as possible, so as to penalize states in which the bit values of the physical qubits representing one logical qubit differ, but there are reasons why setting it too high can be detrimental [13]. The first is that doing so can increase the difficulty of transfering amplitude from a local minimum in which all of the bit values for a component are equal but set to the wrong value. The second is that the device has a finite range of possible couplings, and suffers from significant precision issues, meaning that the noise in the applied coupling strengths is high enough that only about 16 different values can be distinguished. When an Ising problem is sent to the machine, the problem Hamiltonian is automatically rescaled so as to take advantage of the full range. If $J_{\text {int }}$ is set too high, however, when the problem is rescaled, all of the other field strengths become indistinguishable from 0 , and all information specific to the problem is lost. For these reasons, there is a sweet spot for $J_{i n t}$.

For each mapping, a quick parameter sweep was done (not shown) to locate the correct range in which to perform a more detailed evaluation. The tables in Fig. 10 give the median expected total anneal time for $99 \%$ percent success over the benchmark problems under various values of $J_{\text {int }}$ for each of the three mappings, with cell colorings determined from the success probabilities. The table for the direct mapping shows that the optimal value of $J_{\text {int }}$ increases as the problem size and the component size increase, likely because a higher value of $J_{\text {int }}$ is useful for pressuring all of the qubits in the larger components to take on the same value by the end of the computation. The tables for the CNF and time-slice mapping are 

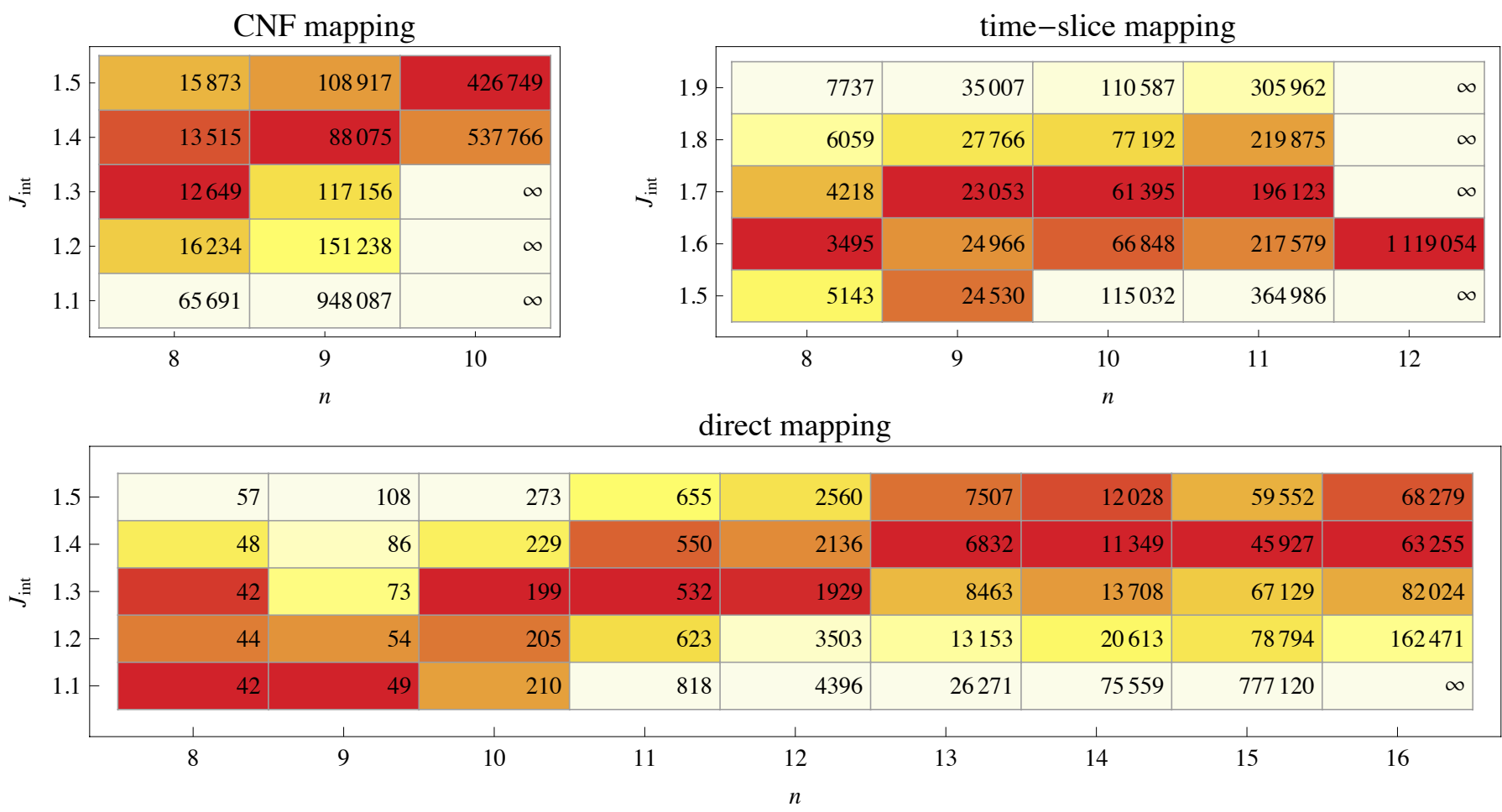

FIG. 10: Performance for different values of $J_{i n t}$ on the three different mappings of scheduling-type planning problems. The numbers are the median expected total anneal time for $99 \%$ percent success over the benchmark set of 100 hard but solvable scheduling-type problems of each size for different values of $J_{\text {int }}$ under the three mappings. The colors were determined from the success probabilities, with each column normalized by the largest value for the given mapping and size. Deep red indicates the largest value, with the colors ranging from orange to yellow to white indicating lower and lower success probabilities.

less conclusive, particularly since only a handful of anneals resulted in a solution for the largest sizes (the last column of each table), but they are consistent with this trend. The time-slice instances benefit from somewhat higher values of $J_{\text {int }}$ than the other two mappings. The same trend was also found in [44].

\section{E. Performance with a simple error correction scheme}

The simple error correction scheme of SecVI does not provide significant improvements in performance on these problems under any of the mappings, as can be seen in Fig. 11. The time-slice results suggest that error correction could provide more significant improvements as the problem size increases. The results on the directly mapped instances, however, show no improvement with error correction over the whole range of problem sizes. The error correction scheme acts only on physical qubits representing the same logical bit that end up taking on opposite values. The reason for these opposite values is likely that there are conflicting constraints with neighboring values. For this reason, majority voting error correction at this level, while it removes any energy penalties resulting from disagreements within the component, will often increase the penalties from constraints involving neighboring logical qubits.

\section{RESULTS ON NAVIGATION-TYPE PLANNING PROBLEMS}

For both the CNF and time-slice mappings of problems from the parametrized family of navigation-type planning problems, the largest problem size that embedded was size 4. For the navigation-specific direct mapping, problems of size 6 embedded reliably, but problems of size 7 no longer embedded. We increased the number of embedding tries, and even asked D-Wave to try to embed these problems for us, but without success. Only problems with fewer than 33 vertices are guaranteed to embed in the 512 vertex $(8,4)$-Chimera graph, so these 49-qubit QUBOs may not embed in the current hardware. While the directly mapped navigation-type planning problems have QUBO graphs that are far from fullyconnected - the number of edges in these graphs goes up linearly with size not quadratically - they appear to be sufficiently connected that it may be impossible to embed any size 7 navigation-type planning problem in the current hardware. The next section explores embedding these problems in larger architectures. For those prob- 

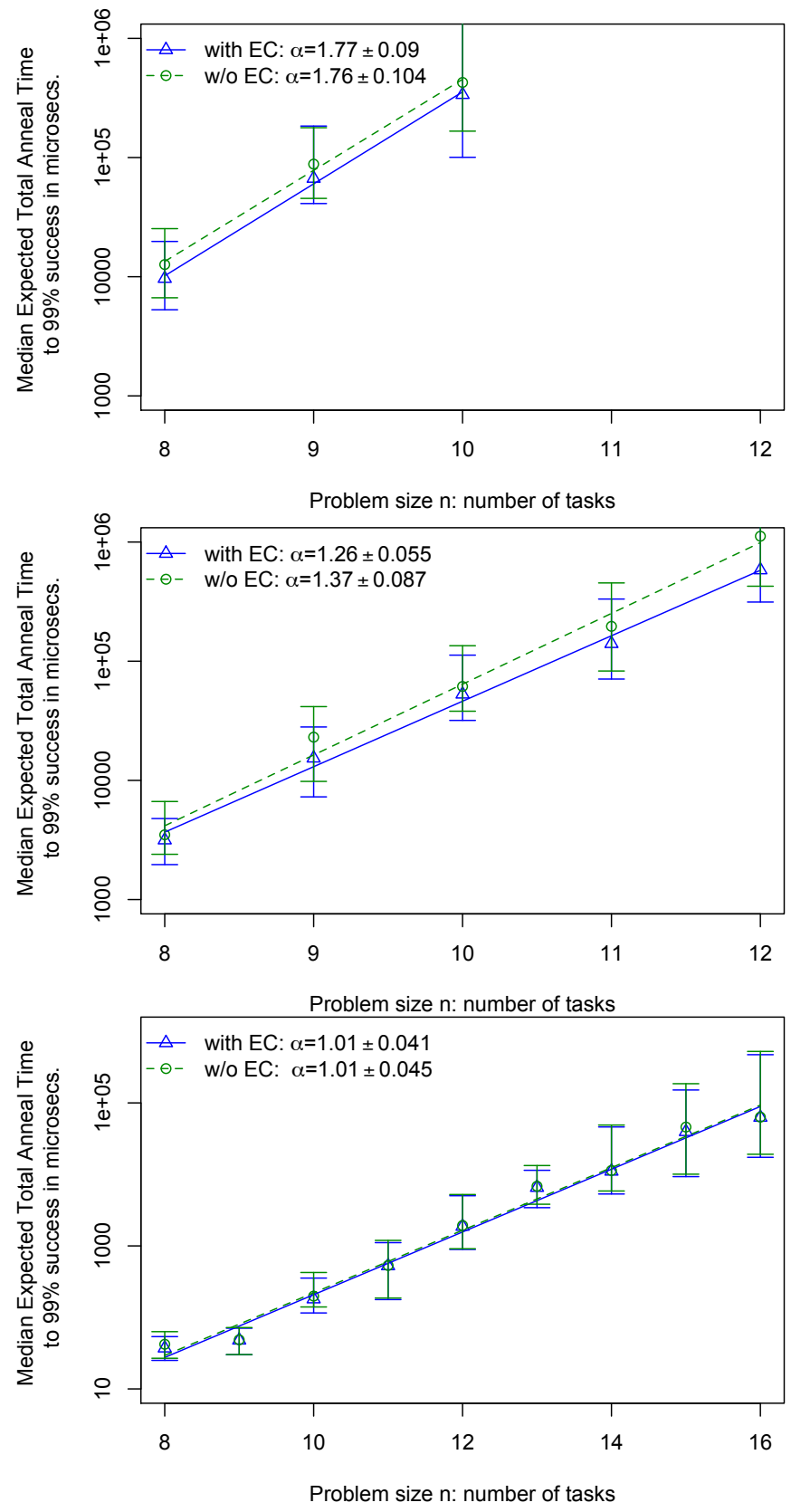

FIG. 11: Comparison of performance with and without simple error correction on the three mappings of scheduling-type planning problems. Median expected total anneal time for $99 \%$ percent success for each mapping, with the best $J_{\text {int }}$ for each size, are shown. CNF mapping (Top): In the corrected case, $J_{\text {int }}$ was $\{1.6,1.5,1.7,1.5,1.6\}$ for problem sizes $\{8,9,10,11,12\}$ respectively. For the uncorrected case, $J_{\text {int }}$ was $\{1.6,1.7,1.7,1.7,1.6\}$ respectively. Time-slice mapping (Middle): In the corrected case, $J_{\text {int }}$ was $\{1.2,1.3,1.3\}$ for problem sizes $\{8,9,10\}$ respectively. For the uncorrected case, $J_{\text {int }}$ was $\{1.2,1.3,1.4\}$ respectively. Direct mapping (Bottom): In the corrected case, $J_{\text {int }}$ was $\{1.1,1.1,1.2,1.3,1.3,1.4,1.4,1.4,1.4\}$ for problem sizes $\{8, \ldots, 12\}$ respectively. For the uncorrected case, $J_{\text {int }}$ was $\{1.1,1.1,1.3,1.3,1.3,1.4,1.4,1.4,1.4\}$ respectively. lems that did embed, the D-Wave machine solved them reliably and quickly.

\section{EMBEDDING IN FUTURE ARCHITECTURES}

We investigated the embeddability of the most embeddable graph, the graph corresponding to the direct mapping to QUBO of the trivial Hamiltonian path problem on the fully connected graph $K_{n}$ of size $n$. This graph, which we call the intersecting-cliques graph $I C_{n, n}$ for reasons that will become apparent shortly, is a subgraph of all the directly mapped navigation problems of its size (and larger) so if this one doesn't embed, none of the others will. The graph is far from fully connected, each of the $n^{2}$ vertices having degree only $2(n-1)$, but the degree does grow in linearly with the size of the problem. The $n^{2}$ vertices in the graph can be partitioned into a set of $n$ cliques each containing $n$ vertices in two different ways. The first clique partition corresponds to the QUBO term enforcing the condition that each site is visited exactly once, and the second to the QUBO term enforcing the condition that only one site is visited at a time. The $n$ vertices in any one of the cliques in the first set are all in different cliques of the second set. This property inspired the name. Diagram of $I C_{5,5}$ and $I C_{3,3}$ are shown in Fig. 12.

We investigated the embeddability of these problems in potential future architectures, specifically $(M, L)$ Chimera graphs 13 in which either the number of unit cells $M^{2}$ or the size of the unit cell $K_{L, L}$ is increased, or both. A deterministic algorithm 27 provides an embedding of any graph with no more than $M L+1$ vertices in an $(M, L)$-Chimera graph, but for graphs that are far from fully connected, this algorithm is usually quite inefficient in the number of qubits used, and in practice many graphs can embed in a smaller Chimera graph than is found by this algorithm. In particular, D-Wave's heuristic embedding software often finds more efficient embeddings.

To investigate the embeddability of these problems in future architectures, we ran D-Wave's heuristic embedding software 11 times per problem on each $(M, L)$ Chimera graph architecture. The heuristic embedding software was run with default parameters, including up to 10 restarts if an embedding is not found on a given try. We recorded the 11 embedding sizes we obtained.

Fig. 14 shows the minimum embedding size for architectures in which the size of the unit cell $K_{L, L}$ remains constant with $L$ remaining at its current value of $L=4$, but the number of unit cells is increased. As can be seen in the figure, increasing the number of unit cells, while retaining the current size of the unit cells $(L=4)$, hardly improves the size of the embeddings of problems that embeddeded in smaller architectures, but does extend the problem size that embeds somewhat.

Fig. 15 shows the minimum embedding size for archi- 

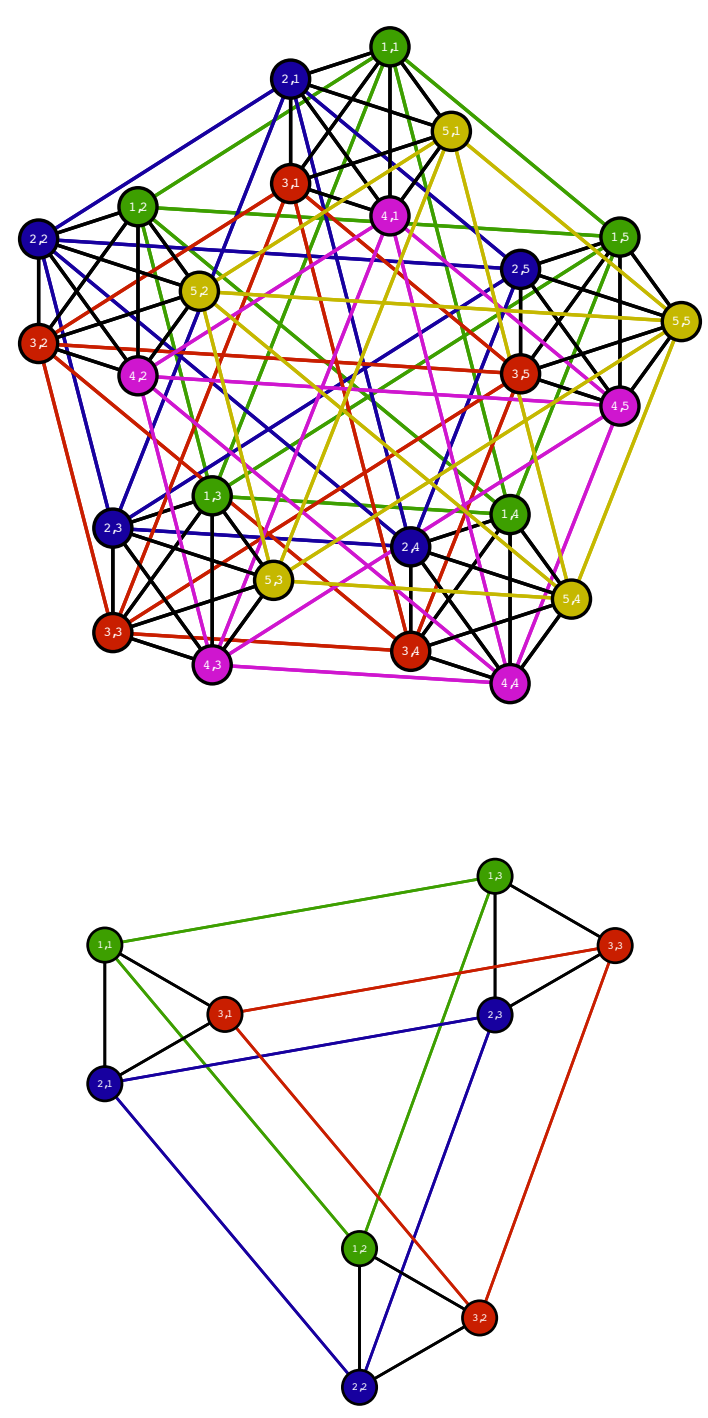

FIG. 12: The intersecting-cliques graphs $I C_{5,5}$ and $I C_{3,3}$. The intersecting-cliques graph $I C_{n, n}$ is a subgraph of all navigation-type problems with $n$ or more sites. It corresponds to the trivial navigation-type problem in which every site is connected directly to every other site. It is the most embeddable of the navigation-type problems, meaning that if it does not embed then neither will any of the other problems with the same number of sites (or more).

tectures in which the size of the unit cell $K_{L, L}$ is increased while the number of unit cells stays constant at its current value $M^{2}=64$. As can be seen in the figure, increasing the size of the unit cells, and thus increasing the local connectivity of the graph, even while the number of unit cells constant, significantly improves the embedding size and the embeddability of these problems.

Fig. 16 explores the embeddability of $I C_{k, k}$ for $k \in$

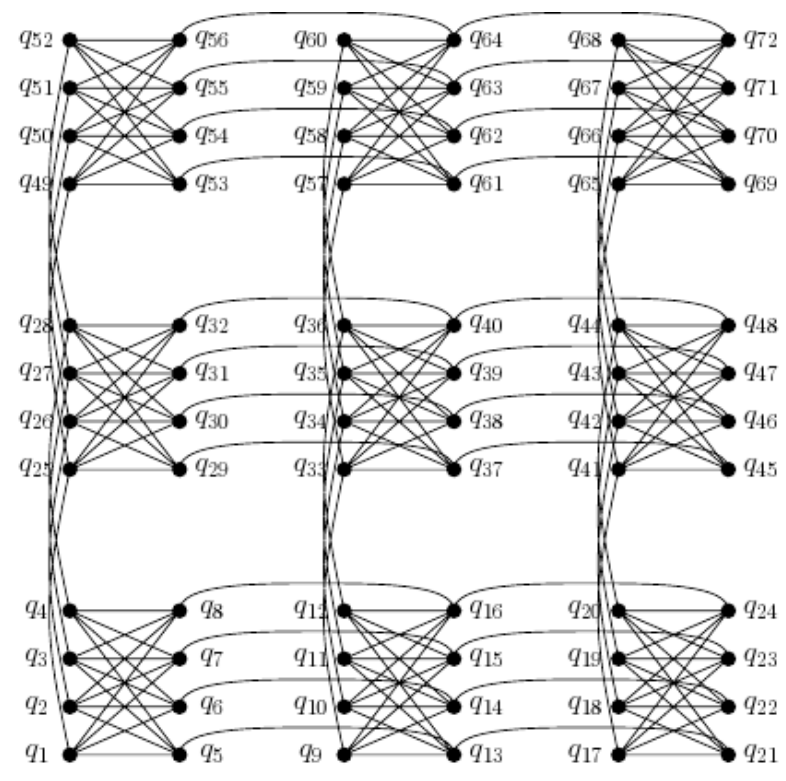

FIG. 13: The (3,4)-Chimera graph. A schematic diagram from [42] of the $(M, L)$-Chimera graph underlying D-Wave's architecture. In the $(3,4)$-Chimera graph shown, there are $M^{2}=9$ unit cells, each of which is a fully-connected bipartite graph $K_{4,4}$ containing $2 L$ qubits. The qubits in the left column of each unit cell are connected to the analogous qubits in the unit cells above and below (add or subtract $M L=24$ to the index) and the qubits in the right column of each unit cell are connected to the analogous qubits in the unit cells to the right and left (add or subtract $2 L=8$ to the index). The DWave Two used in the experiments has a $(8,4)$-Chimera graph architecture, but with 3 broken qubits that are not used.

$\{7,8,9,10\}$ for the full range of architectures with $M \in$ $\{8, \ldots, 16\}$ and $L \in\{4, \ldots, 10\}$. The embedding software runs in seconds for problem sizes less than 7 , but increases rapidly after that, with the 11 embedding trials taking $30-45$ minutes for some of the larger problem sizes. For this reason, we did not explore problem sizes larger than 10.

By the end of 2014, a 1024 qubit D-Wave machine should be available at NASA, and sometime in 2015, a 2048 machine should be available. Unfortunately, if these qubit numbers are achieved by simply increasing the number of unit cells, rather than increasing the connectivity of the unit cells, or moving to a different architecture altogether, it looks likely that only navigationtype planning problems of size less than 10 will be able to be run on such machines due to the difficulty embedding larger instances in these hardware graphs. Alternative embedding strategies could improve these results, but in the near-term, scheduling-type planning problems appear more suitable than navigation-type planning problems for these near-term Chimera architectures with $K_{4,4}$ unit cell. For real world planning applications that combine both navigation and scheduling aspects, the design of new hardware architectures that will overcome the 


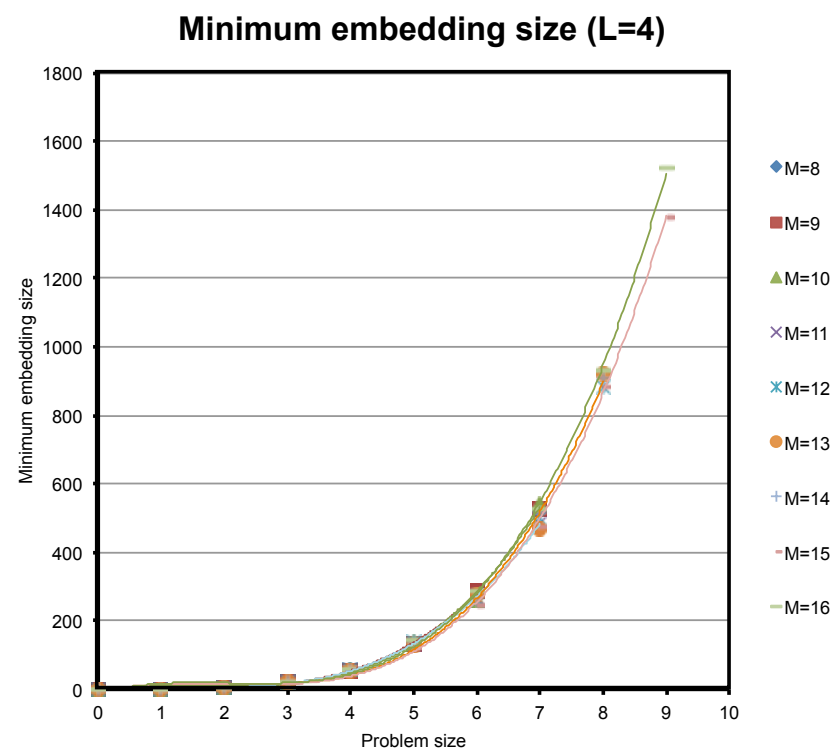

FIG. 14: $I C_{k, k}$ embedding sizes with increasing number of $K_{4,4}$ unit cells. The embedding sizes of the intersectingcliques graph $I C_{k, k}$ for $k \leq 10$ in $(M, 4)$-Chimera graphs for $M \in\{8, \ldots$,$\} . Increasing the number of unit cells, M^{2}$, does little to improve the embedding sizes of graphs that embedded in a smaller architecture, but does extend somewhat the range of $k$ over which $I C_{k, k}$ embeds reliably within 11 runs of the DWave heuristic embedding software with default parameters. The points for sizes 0 and 1 were put in manually. The rest reflect runs of D-Wave's heuristic embedding software.

\section{Minimum embedding size $(M=8)$}

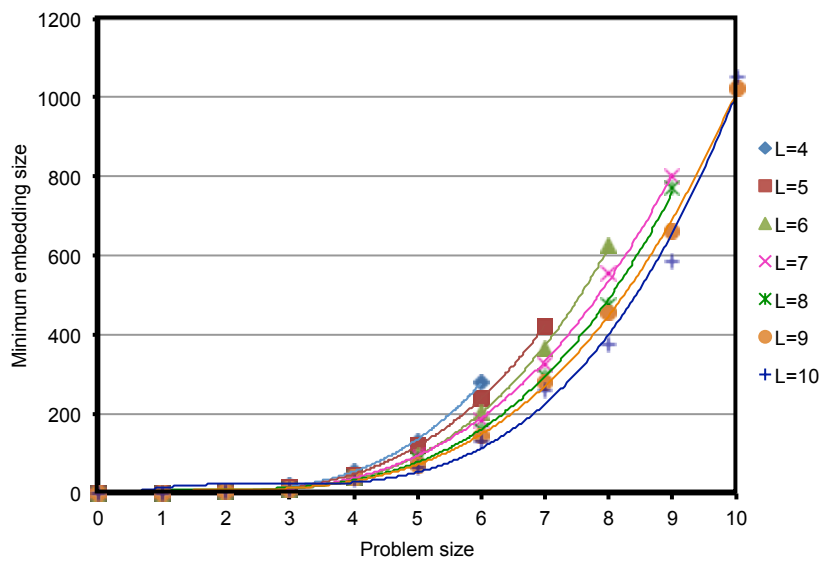

FIG. 15: $I C_{k, k}$ embedding sizes with increasing unit cell size. The embedding sizes of the intersecting-cliques graph $I C_{k, k}$ for $k \leq 10$ in $(8, L)$-Chimera graphs for $L \in$ $\{4, \ldots, 10\}$. Increasing the size of the $K_{L, L}$ unit cells, and thereby the local connectivity of the hardware graph, while keeping the number of unit cells constant, enables improved embedding sizes of graphs that embedded in a smaller architecture, as well as extending the range of $k$ for which $I C_{k, k}$ embeds reliably.

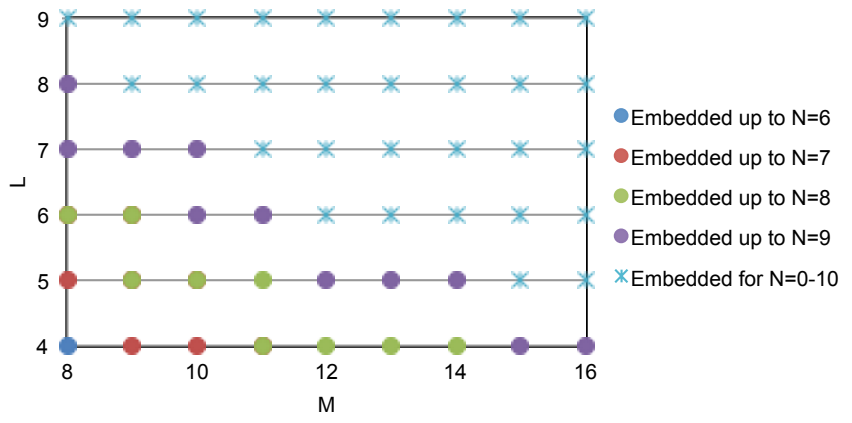

FIG. 16: Embedding of $I C_{k, k}$ graphs in $(M, L)$-Chimera graphs. Embedding of $I C_{k, k}$ for $k \leq 10$ in $(M, L)$-Chimera graphs for $M \in\{8, \ldots, 16\}$ and $L \in\{4, \ldots, 8\}$. Dots show the largest size problem which embedded in the given architecture in 11 runs of the D-Wave heuristic embedding software with default parameters. Crosses indicate that the next largest size problem $(n=11)$ was not run, so we do not have data to indicate whether or not it would have embedded in the given Chimera architecture.

programming bottleneck presented by embedding these problems is critical.

While the scheduling-type planning problems embed better than the navigation-type planning problems, for both of the general mappings, by around problem size 15 or 16, the heuristic embedding software has difficulty embedding these problems. For the direct map instances, the heuristic embedding software had no problems embedding instances up to problem size 17 , begins to have occasional difficulties at problem size 18 , and has serious difficulties embedding most of the problems by size 22 .

We finish the discussion of embedding in future architectures with an analysis of the embeddability of the simplest solvable scheduling-type planning problem, the one that corresponds to a completely disconnected graph of $n$ vertices. As in the navigation case, its QUBO graph is a subgraph of the QUBO graph for all of the schedulingtype problems, so if it doesn't embed, none will. The analysis is easier than in the navigation case, and can be done by hand. The QUBO graph consists of $n$ triangles (we are considering the 3 time slot case). Since $k$ triangles can embed in a $K_{2 k, 2 k}$ unit cell, an $(M, 2 k)$ Chimera architecture supports the embedding of the simplest $k M^{2}$-task problem. Since 4 vertices are required to embed a triangle in Chimera graph architecture, this embedding is optimal. As an example, the simplest problem with 128 tasks embeds in a 512 qubit machine, and the 512 task problem embeds in the 2048 machine projected for 2015. In addition, preliminary tests show that typical scheduling-type problems of size well past 50 will embed in the 2048 architecture. 


\section{CONCLUSIONS AND FUTURE WORK}

We have studied the effectiveness of a quantum annealer in solving small instances within families of hard operational planning problems under various mappings and embeddings. From these results we derive insights useful for the programming and design of future quantum annealers: problem choice, the mapping used, properties of the embedding, and annealing profile all matter, each significantly affecting the performance. While this initial study did not produce results competitive with state-of-the-art classical approaches, higher quality qubits, shorter annealing times, better precision, greater hardware connectivity, improved mappings and embeddings, and alternative annealing profiles will all contribute to improved results.

In future work, we will experiment with other mappings, including experimenting with different weightings of penalty terms in the QUBO funtion and other translations of the problem to CNF form, and also other prob- lems from operational planning, such as job shop scheduling. We will also build on this work, exploring more embeddings per problem to sort out how much of the variation in runtime is due to the variation in the difficulty of the problems themselves versus how much is due to properties of the embedding independent of the problem. We will perform statistical analysis of the correlation between a richer set of embedding properties and expected total annealing time in order to give insight into better metrics for evaluating embeddings of QUBO problems for quantum annealing.

\section{Acknowledgements}

The authors are grateful to Jeremy Frank, Alejandro Perdomo-Ortiz, Ross Beyer, Sergey Knysh, Chris Henze, and Itay Hen for helpful discussions, and to DWave for technical support and for discussions related to the calibration issue and our results before and after.
[1] Achlioptas, D. and Friedgut, E. (1999). A sharp threshold for k-colorability. Random Structures and Algorithms, 14(1):63-70.

[2] Achlioptas, D. and Moore, C. (2003). Almost all graphs with average degree 4 are 3-colorable. Journal of Computer and System Sciences, 67(2):441-471.

[3] Babbush, R., Love, P. J., and Aspuru-Guzik, A. (2013a). Adiabatic quantum simulation of quantum chemistry. arXiv:1311.3967.

[4] Babbush, R., O'Gorman, B., and Aspuru-Guzik, A. (2013b). Resource efficient gadgets for compiling adiabatic quantum optimization problems. Annalen der Physik, 525(10-11):877-888.

[5] Babbush, R., Perdomo-Ortiz, A., O'Gorman, B., Macready, W., and Aspuru-Guzik, A. (2012). Construction of energy functions for lattice heteropolymer models: A case study in constraint satisfaction programming and adiabatic quantum optimization. arXiv:1211.3422.

[6] Blum, A. and Furst, M. (1997). Planning through planning graph analysis. Artificial Intelligence Journal, 90:281-330.

[7] Boixo, S., Albash, T., Spedalieri, F. M., Chancellor, N., and Lidar, D. A. (2013). Experimental signature of programmable quantum annealing. Nature communications, 4.

[8] Boixo, S., Rønnow, T. F., Isakov, S. V., Wang, Z., Wecker, D., Lidar, D. A., Martinis, J. M., and Troyer, M. (2014). Evidence for quantum annealing with more than one hundred qubits. Nature Physics, 10(3):218-224.

[9] Boros, E. and Hammer, P. L. (2002). Pseudo-boolean optimization. Discrete applied mathematics, 123(1):155225.

[10] Cai, J., Macready, B., and Roy, A. (2014). A practical heuristic for finding graph minors. arXiv:1406:2741.

[11] Cheeseman, P., Kanefsky, B., and Taylor, W. M. (1991). Where the really hard problems are. In IJCAI, volume 91, pages 331-337.
[12] Chien, S., Johnston, M., Frank, J., Giuliano, M., Kavelaars, A., Lenzen, C., Policella, N., and Verfailie, G. (2012). A generalized timeline representation, services, and interface for automating space mission operations. In 12th International Conference on Space Operations.

[13] Choi, V. (2008). Minor-embedding in adiabatic quantum computation: I. the parameter setting problem. Quantum Information Processing, 7(5):193-209.

[14] Coja-Oghlan, A. (2013). Upper-bounding the k-colorability threshold by counting covers. arXiv:1305.0177.

[15] Culberson, J., Beacham, A., and Papp, D. (1995). Hiding our colors. In Proceedings of the CP95 Workshop on Studying and Solving Really Hard Problems, pages 31-42.

[16] Das, A. and Chakrabarti, B. K. (2008). Colloquium: Quantum annealing and analog quantum computation. Rev. Mod. Phys., 80:1061-1081.

[17] Dubois, O. and Mandler, J. (2002). On the non-3colourability of random graphs. arXiv:math/0209087.

[18] Farhi, E., Goldstone, J., Gutmann, S., and Sipser, M. (2000). Quantum computation by adiabatic evolution. arXiv:quant-ph/0001106.

[19] Fikes, R. E. and Nilsson, N. J. (1972). STRIPS: A new approach to the application of theorem proving to problem solving. Artificial intelligence, 2(3):189-208.

[20] Ghallab, M., Nau, D., and Traverso, P. (2004). Automated planning: theory $\&$ practice. Elsevier.

[21] Helmert, M. (2003). Complexity results for standard benchmark domains in planning. Artificial Intelligence Journal, pages 219-262.

[22] Hoffmann, J. (2005). Where ignoring delete lists works: Local search topology in planning benchmarks. Journal of Artificial Intelligence Research, 24:685-758.

[23] Johnson, M., Amin, M., Gildert, S., Lanting, T., Hamze, F., Dickson, N., Harris, R., Berkley, A., Johansson, J., Bunyk, P., et al. (2011a). Quantum annealing with manufactured spins. Nature, 473(7346):194-198. 
[24] Johnson, M. W., Amin, M. H. S., Gildert, S., and et al. (2011b). Quantum annealing with manufactured spins. Nature, 473:194-198.

[25] Kassal, I., Whitfield, J. D., Perdomo-Ortiz, A., Yung, M.H., and Aspuru-Guzik, A. (2010). Simulating chemistry using quantum computers. arXiv:1007.2648.

[26] Kautz, H. (2004). Satplan04: Planning as satisfiability. Working Notes on the Fourth International Planning Competition (IPC-2004), pages 44-45.

[27] Klymko, C., Sullivan, B. D., and Humble, T. S. (2014). Adiabatic quantum programming: minor embedding with hard faults. Quantum information processing, 13(3):709-729.

[28] Komlós, J. and Szemerédi, E. (1983). Limit distribution for the existence of Hamiltonian cycles in a random graph. Discrete Mathematics, 43(1):55-63.

[29] Long, D. and Fox, M. (2003). The 3rd international planning competition: Results and analysis. J. Artif. Intell. Res.(JAIR), 20:1-59.

[30] Lucas, A. (2013). Ising formulations of many NP problems. arXiv:1302.5843.

[31] Nielsen, M. and Chuang, I. L. (2001). Quantum Computing and Quantum Information. Cambridge University Press, Cambridge.

[32] O'Gorman, B., Perdomo-Ortiz, A., Babbush, R., Aspuru-Guzik, A., and Smelyanskiy, V. (2014). Bayesian network structure learning using quantum annealing. In submission.

[33] Perdomo-Ortiz, A., Dickson, N., Drew-Brook, M., Rose, G., and Aspuru-Guzik, A. (2012). Finding low-energy conformations of lattice protein models by quantum annealing. Scientific reports, 2.

[34] Perdomo-Ortiz, A., Fluegemann, J., , Narasimhan, S., Smelyanskiy, V., and Biswas, R. (2014a). A quantum approach to diagnosis of multiple faults in electrical power systems. 5th IEEE international Conference on Space Mission Challenges for Information Technology. [To appear.].

[35] Perdomo-Ortiz, A., Fluegemann, J., Narasimhan, S., Biswas, R., and Smelyanskiy, V. (2014b). Programming and solving real-world applications on a quantum annealing device. arXiv:1406.7601.

[36] Pudenz, K. L., Albash, T., and Lidar, D. A. (2014). Error-corrected quantum annealing with hundreds of qubits.

[37] Rieffel, E. G. and Polak, W. (2011). A Gentle Introduction to Quantum Computing. MIT Press, Cambridge,
MA.

[38] Rieffel, E. G., Venturelli, D., Hen, I., Do, M., and Frank, J. (2014). Parametrized families of hard planning problems from phase transitions. Proceedings of the Twenty-Eighth AAAI Conference on Artificial Intelligence (AAAI-14)[To appear].

[39] Rønnow, T. F., Wang, Z., Job, J., Boixo, S., Isakov, S. V., Wecker, D., Martinis, J. M., Lidar, D. A., and Troyer, M. (2014). Defining and detecting quantum speedup. arXiv:1401.2910.

[40] Shin, S. W., Smith, G., Smolin, J. A., and Vazirani, U. (2014a). Comment on "Distinguishing classical and quantum models for the D-Wave device". arXiv:1404.6499.

[41] Shin, S. W., Smith, G., Smolin, J. A., and Vazirani, U. (2014b). How "quantum" is the D-Wave machine? arXiv:1401.7087.

[42] Smelyanskiy, V. N., Rieffel, E. G., Knysh, S. I., Williams, C. P., Johnson, M. W., Thom, M. C., Macready, W. G., and Pudenz, K. L. (2012). A near-term quantum computing approach for hard computational problems in space exploration. arXiv:1204.2821.

[43] Smolin, J. A. and Smith, G. (2013). Classical signature of quantum annealing. arXiv:1305.4904.

[44] Venturelli, D., Mandra, S., Knysh, S., OGorman, B., Biswas, R., and Smelyanskiy, V. (2014). Quantum optimization of fully-connected spin glasses. arXiv:1406.7553.

[45] Vinci, W., Albash, T., Mishra, A., Warburton, P. A., and Lidar, D. A. (2014). Distinguishing classical and quantum models for the D-Wave device. arXiv:1403.4228.

[46] Wang, L., Rønnow, T. F., Boixo, S., Isakov, S. V., Wang, Z., Wecker, D., Lidar, D. A., Martinis, J. M., and Troyer, M. (2013a). Comment on "Classical signature of quantum annealing".

[47] Wang, Z., Boixo, S., Albash, T., and Lidar, D. (2013b). Benchmarking the D-Wave adiabatic quantum optimizer via 2D-Ising spin glasses. In APS Meeting Abstracts, volume 1, page 27005 .

[48] Wang, Z., Job, J., Isakov, S. V., Boixo, S., Lidar, D., Martinis, J., Troyer, M., et al. (2014a). Probing for quantum speedup on D-Wave Two. Bulletin of the American Physical Society.

[49] Wang, Z., Job, J., Troyer, M., Lidar, D. A., et al. (2014b). Performance of quantum annealing on random Ising problems implemented using the D-Wave Two. Bulletin of the American Physical Society. 